\title{
Pseudoduality In Supersymmetric Sigma Models
}

\author{
Mustafa Sarisaman* \\ Department of Physics \\ University of Miami \\ P.O. Box 248046 \\ Coral Gables, FL 33124 USA
}

Monday, April 27, 2009

\begin{abstract}
We study the pseudoduality transformation in supersymmetric sigma models. We generalize the classical construction of pseudoduality transformation to supersymmetric case. We perform this both by component expansion method on manifold $\mathbb{M}$ and by orthonormal coframe method on manifold $S O(\mathbb{M})$. The component expansion method yields the result that pseudoduality tranformation is not invertible at all points and occurs from all points on one manifold to only one point where riemann normal coordinates are valid on the second manifold. Torsion of the sigma model on $\mathbb{M}$ must vanish while it is nonvanishing on $\tilde{\mathbb{M}}$, and curvatures of the manifolds must be constant and the same. In case of super WZW sigma models pseudoduality equations result in three different pseudoduality conditions; flat space, chiral and antichiral pseudoduality.
\end{abstract}

*msarisaman@physics.miami.edu 


\section{Introduction}

This model consists of both bosons and fermions, and they are transformed into each other by supersymmetry transformation. It improves the short distance behaviour of quantum theories and gives a beautiful solution to the hierarchy problem. Supersymmetric sigma models have a rich geometrical structure. It has been shown [1] that target space of $N=1$ sigma models is a (pseudo-)Riemannian manifold, $N=2$ is the Kähler manifold and $N=$ 4 is the hyper-Kähler manifold. Sigma models based on manifolds with torsion [2] have chiral supersymmetry in which the number of left handed supersymmetries differs from the number of right handed supersymmetries.

There is an interesting duality transformation proposed by authors [3, 4, 5, 6], which is called as "pseudoduality". By contrast with usual duality transformations this "on shell duality" transformation is not canonical, and maps solutions of the equations of motion of the "pseudodual" models. We will use the term pseudodual when there is a pseudoduality transformation between different models. It is pointed out that this transformation preserves the stress energy tensor [6].

In [6], pseudoduality in classical sigma models was extensively discussed, and in this paper we are going to analyze pseudoduality transformation of supersymmetric extension of classical sigma models. We will focus on $(1,0)$ and $(1,1)$ real supersymmetric sigma models in two dimensions, and find the required conditions which supersymmetry constrains the target space and following results for pseudoduality. We will refer to references [1, 7, 8, 9, 10] about supersymmetry and superspace constructions.

We use the superspace coordinates $\left(\sigma^{ \pm}, \theta^{ \pm}\right)$, where the bosonic coordinates $\sigma^{ \pm}=\tau \pm \sigma$ are the usual lightcone coordinates in two-dimensional Minkowski space, and the fermionic coordinates $\theta^{ \pm}$are the Grassmann numbers. The supercovariant derivatives are

$$
D_{ \pm}=\partial_{\theta^{ \pm}}+i \theta^{ \pm} \partial_{ \pm}
$$

and the supercharges generating supersymmetry are

$$
Q_{ \pm}=\partial_{\theta^{ \pm}}-i \theta^{ \pm} \partial_{ \pm}
$$

and it follows that

$$
Q_{ \pm}^{2}=-i \partial_{ \pm} \quad D_{ \pm}^{2}=i \partial_{ \pm}
$$


and all other anticommutations vanish. The scalar superfields in components have the form

$$
X(\sigma, \theta)=x(\sigma)+\theta^{+} \psi_{+}(\sigma)+\theta^{-} \psi_{-}(\sigma)+\theta^{+} \theta^{-} F(\sigma)
$$

where $x: \Sigma \rightarrow M, \psi_{ \pm}$are the two dimensional Majorana spinor fields, and $F$ is the auxiliary real scalar field.

\section{Pseudoduality in Heterotic Sigma Models}

This model [1, 2, 11, 12, 13, 14 is enlarging the spacetime $\Sigma$ in the classical case to the superspace $\Xi^{1,0}$ by adding a Grassmann degree of freedom. Hence the sigma model is the map consisting of a scalar $x$ and a fermion $\psi_{+}$. This case has one left-handed supercharge $Q_{+}$, and does not contain any righthanded supercharge $Q_{-}$. The supersymmetry algebra will be

$$
\left\{Q_{+}, Q_{+}\right\}=2 i P_{+}
$$

where $\{$,$\} denotes anticommutation, and P_{+}=-\partial_{+}$as can be checked from (3). The supersymmetry transformations generated by $Q_{+}$will be

$$
\begin{aligned}
\delta_{\epsilon} x(\sigma) & =\epsilon_{-} \psi_{+}(\sigma) \\
\delta_{\epsilon} \psi_{+}(\sigma) & =i \epsilon_{-} \partial_{+} x(\sigma)
\end{aligned}
$$

Hence the fermion $\psi_{+}$can be thought of as the superpartner of the boson $x$. In what follows we will examine pseudoduality transformations between supermanifolds $\mathbb{M} 11$ and $\tilde{\mathbb{M}}$ using components first, and then probe how it behaves when lifted to orthonormal coframe bundles $S O(\mathbb{M}) 2$ and $S O(\tilde{\mathbb{M}})$. We emphasize that pseudoduality is defined between superspaces $z$ which are the pullbacks of the manifols $\mathbb{M}$ and $\tilde{\mathbb{M}}$ in case of components, and $S O(\mathbb{M})$ and $S O(\tilde{\mathbb{M}})$ in case of orthonormal coframe method. This is implicitly intended in our calculations.

\subsection{Components}

In this case the superfield $X$ has the form

$$
X=x(\sigma)+\theta^{+} \psi_{+}(\sigma)
$$

\footnotetext{
${ }^{1} \mathbb{M}$ is the target space in which supersymmetric sigma models is defined.

${ }^{2} S O(\mathbb{M})=\mathbb{M} \times S O(n)$.
} 
where $X: \Xi^{1,0} \rightarrow \mathbb{M}$, and $\Xi^{1,0}=\left(\sigma^{+}, \sigma^{-}, \theta^{+}\right)$. The real grassmann coordinate $\theta^{+}$is anticommuting and $\left(\theta^{+}\right)^{2}=0$. We will assume that target space has torsion $\mathrm{H}$, which is introduced into the action by a Wess-Zumino term. Reparametrization invariant action defined on a Riemannian manifold $\mathbb{M}$ with metric $G_{i j}$, standard connection $\Gamma_{j k}^{i}$ and antisymmetric two-form $B_{i j}$ can be written as

$$
S=\int d^{2} \sigma d \theta\left(G_{i j}+B_{i j}\right) D_{+} X^{i} \partial_{-} X^{j}
$$

We may write similar expressions for manifold $\tilde{\mathbb{M}}$ using expressions with tilde. Since we want to write down pseudoduality transformations between two manifolds, we need to find out the equations of motions from action (6). If we write this action in terms of bosonic coordinates of superspace only, we obtain our original classical action plus fermionic terms. After expanding $G_{i j}$ and $B_{i j}$ in the first order terms and integrating this action under $d \theta$ gives the following

$$
S=\int d^{2} \sigma\left[i\left(g_{i j}+b_{i j}\right) \partial_{+} x^{i} \partial_{-} x^{j}-g_{i j} \psi_{+}^{i} \nabla_{-}^{(-)} \psi_{+}^{j}\right]
$$

where $\nabla_{-}^{(-)} \psi_{+}^{j}=\nabla_{-} \psi_{+}^{j}-H_{k l}^{j} \psi_{+}^{k} \partial_{-} x^{l}$ and $\nabla_{-} \psi_{+}^{j}=\partial_{-} \psi_{+}^{j}+\Gamma_{k l}^{j} \psi_{+}^{k} \partial_{-} x^{l}$, and $H_{i j k}=\frac{1}{2}\left(\partial_{i} b_{j k}+\partial_{j} b_{k i}+\partial_{k} b_{i j}\right)$. Equations of motion following from the action (71) are

$$
\begin{aligned}
\nabla_{-}^{(-)} \psi_{+}^{i} & =0 \\
\square x^{k} & =i \hat{R}_{l i j}^{k} \psi_{+}^{i} \psi_{+}^{j} \partial_{-} x^{l}
\end{aligned}
$$

where $\square x^{k}=\nabla_{+}^{(+)} \partial_{-} x^{k}+\nabla_{-}^{(-)} \partial_{+} x^{k}$, and the generalized curvature is defined as

$$
\hat{R}_{i j k l}=R_{i j k l}-D_{k} H_{i j l}+D_{l} H_{i j k}+H_{i k n} H_{l j}^{n}-H_{j k n} H_{l i}^{n}
$$

We can write the Pseudoduality transformations as follows

$$
\begin{aligned}
D_{+} \tilde{X}^{i} & =+\mathcal{T}_{j}^{i} D_{+} X^{i} \\
\partial_{-} \tilde{X}^{i} & =-\mathcal{T}_{j}^{i} \partial_{-} X^{j}
\end{aligned}
$$

where $\mathcal{T}$ is the transformation matrix, and is a function of superfield $X$. Since superfield depends on $\sigma$ and $\theta^{+}$, we may say that $\mathcal{T}$ is a function of $\sigma$ 
and $\theta^{+}$. We let $\mathcal{T}(\sigma, \theta)=T(\sigma)+\theta^{+} N(\sigma)$. Splitting pseudoduality equations into the fermionic and bosonic parts leads to the following set of equations

$$
\begin{aligned}
\tilde{\psi}_{+}^{i}(\sigma) & =+T_{j}^{i}(\sigma) \psi_{+}^{j}(\sigma) \\
\partial_{-} \tilde{\psi}_{+}^{i}(\sigma) & =-T_{j}^{i}(\sigma) \partial_{-} \psi_{+}^{j}(\sigma)-N_{j}^{i}(\sigma) \partial_{-} x^{j}(\sigma) \\
\partial_{+} \tilde{x}^{i}(\sigma) & =+T_{j}^{i}(\sigma) \partial_{+} x^{j}(\sigma)-i N_{j}^{i}(\sigma) \psi_{+}^{j}(\sigma) \\
\partial_{-} \tilde{x}^{i}(\sigma) & =-T_{j}^{i}(\sigma) \partial_{-} x^{j}(\sigma)
\end{aligned}
$$

We see that the component $T$ is responsible for the classical transformation which does not change the type of field, while $N$ contributes to the fermionic degree of transformation which transforms bosonic fields to fermionic ones, and vice versa. Before finding pseudodual expressions it is worth to obtain constraint relations. We take $\partial_{-}$of (13) and set equal to (14), and then use the equation of motion (8) to obtain

$$
N_{k}^{i}=-\left[M_{l k}^{i}+2 T_{j}^{i}\left(H_{l k}^{j}-\Gamma_{l k}^{j}\right)\right] \psi_{+}^{l}
$$

where we define $\partial_{k} T_{l}^{i}=M_{l k}^{i}$. Now taking $\partial_{+}$of (16) and setting equal to $\partial_{-}$ of (15) followed by using equations of motion (8) and (9) yields

$$
\begin{aligned}
& {\left[2 T_{k}^{i}\left(H_{m n}^{k}-\Gamma_{m n}^{k}\right)+2 M_{(m n)}^{i}\right] \partial_{+} x^{m} \partial_{-} x^{n}+i T_{k}^{i} \hat{R}_{m i j}^{k} \psi_{+}^{i} \psi_{+}^{j} \partial_{-} x^{m}} \\
& =i N_{k}^{i}\left(H_{m n}^{k}-\Gamma_{m n}^{k}\right) \psi_{+}^{m} \partial_{-} x^{n}+i\left(\partial_{-} N_{k}^{i}\right) \psi_{+}^{k}
\end{aligned}
$$

where $M_{(m n)}^{i}$ represents the symmetric part of $M_{m n}^{i}$. Real part of this equation gives

$$
T_{k}^{i}\left(H_{m n}^{k}-\Gamma_{m n}^{k}\right)+2 M_{(m n)}^{i}=0
$$

which implies that

$$
\begin{aligned}
H_{m n}^{k} & =0, \\
M_{(m n)}^{i} & =T_{k}^{i} \Gamma_{m n}^{k}
\end{aligned}
$$

Substituting these results into (17) leads to

$$
N_{k}^{i}=M_{k m}^{i} \psi_{+}^{m}
$$

Complex part of (18) together with (20), (21) and (22) gives the following equation

$$
\partial_{n} M_{[m j]}^{i}=T_{k}^{i} R_{n j m}^{k}+2 M_{[k j]}^{i} \Gamma_{m n}^{k}
$$

where $M_{[m j]}^{i}$ denotes the antisymmetric part of $M_{m j}^{i}$. Solution of this equation gives the result for $T$. 


\subsubsection{Riemann Normal Coordinates}

Before we attempt to find the general (global) solution for the equation (23), it is interesting to find the special solution where Riemann Normal coordinates [15, 16, 17] are used in both models. In these coordinates solution is expanded around a point (call this point as $p$ on $M$, and $\tilde{p}$ on $\tilde{M}$ ) which Christoffel's symbols vanish. Curvature tensor $R$ is the curvature of the point $p$, and constant. (21) implies that $M_{j m}^{i}=-M_{m j}^{i}$, and hence, equation (23) is reduced to

$$
\partial_{n} M_{m j}^{i}=T_{k}^{i} R_{n j m}^{k}
$$

After integration we get

$$
M_{m j}^{i}=M_{m j}^{i}(0)+\int T_{k}^{i} R_{n j m}^{k} d x^{n}
$$

and since $T_{m}^{i}=T_{m}^{i}(0)+\int M_{m j}^{i} d x^{j}$, we finally obtain

$T_{m}^{i}=T_{m}^{i}(0)+M_{m j}^{i}(0) x^{j}+T_{k}^{i}(0) R_{n j m}^{k} \int x^{n} d x^{j}+M_{k l}^{i}(0) R_{n j m}^{k} \int d x^{j} \int x^{l} d x^{n}+H . O$.

and

$$
M_{m j}^{i}=M_{m j}^{i}(0)+T_{k}^{i}(0) R_{n j m}^{k} x^{n}+M_{k l}^{i}(0) R_{n j m}^{k} \int x^{l} d x^{n}+\text { H.O. }
$$

and also using (22) we find

$$
N_{k}^{i}=M_{k m}^{i}(0) \psi_{+}^{m}+T_{j}^{i}(0) R_{n m k}^{j} \psi_{+}^{m} x^{n}+M_{j l}^{i}(0) R_{n m k}^{j} \psi_{+}^{m} \int x^{l} d x^{n}+H . O .
$$

We choose the initial condition $T_{m}^{i}(0)=\delta_{m}^{i}$. Hence Pseudoduality relations (13) - (16) up to the second order in $x$ can be written as

$$
\begin{aligned}
\tilde{\psi}_{+}^{i} & =\psi_{+}^{i}+M_{j k}^{i}(0) \psi_{+}^{j} x^{k}+R_{n k j}^{i} \psi_{+}^{j} \int x^{n} d x^{k}+H . O . \\
\partial_{-} \tilde{\psi}_{+}^{i} & =-M_{j m}^{i}(0) \psi_{+}^{m} \partial_{-} x^{j}-R_{n m j}^{i} \psi_{+}^{m} x^{n} \partial_{-} x^{j}+H . O . \\
\partial_{+} \tilde{x}^{i} & =\partial_{+} x^{i}+M_{j k}^{i}(0) x^{k} \partial_{+} x^{j}-i M_{j m}^{i}(0) \psi_{+}^{m} \psi_{+}^{j}-i R_{n m j}^{i} \psi_{+}^{m} \psi_{+}^{j} x^{n} \\
& +R_{n k j}^{i} \partial_{+} x^{j} \int x^{n} d x^{k}-i M_{k l}^{i}(0) R_{n m j}^{k} \psi_{+}^{m} \psi_{+}^{j} \int x^{l} d x^{n}+H . O . \\
\partial_{-} \tilde{x}^{i} & =-\partial_{-} x^{i}-M_{j k}^{i}(0) x^{k} \partial_{-} x^{j}-R_{n l j}^{i} \partial_{-} x^{j} \int x^{n} d x^{l}+H . O .
\end{aligned}
$$


Using the equation of motion (8) for tilde, i.e. $\partial_{-} \tilde{\psi}_{+}^{i}=\tilde{H}_{j k}^{i} \tilde{\psi}_{+}^{j} \partial_{-} \tilde{x}^{k}$, and combining with (24) and (27) we find

$\partial_{-} \tilde{\psi}_{+}^{i}=-\tilde{H}_{m j}^{i} \psi_{+}^{m} \partial_{-} x^{j}-\tilde{H}_{m k}^{i} M_{j n}^{k}(0) \psi_{+}^{m} x^{n} \partial_{-} x^{j}-\tilde{H}_{k j}^{i} M_{m n}^{k}(0) \psi_{+}^{m} x^{n} \partial_{-} x^{j}+H . O$.

A comparison of equation (25) with equation (28) gives

$$
\begin{aligned}
\tilde{H}_{m j}^{i} & =M_{j m}^{i}(0) \\
R_{n m j}^{i} & =M_{k m}^{i}(0) M_{j n}^{k}(0)+M_{j k}^{i}(0) M_{m n}^{k}(0)
\end{aligned}
$$

Now we see that equation (9) with tilde is written as $\partial_{+-}^{2} \tilde{x}^{i}=\tilde{H}_{j k}^{i} \partial_{+} \tilde{x}^{j} \partial_{-} \tilde{x}^{k}+$ $\frac{i}{2} \hat{\tilde{R}}_{j k l}^{i} \tilde{\psi}_{+}^{k} \tilde{\psi}_{+}^{l} \partial_{-} \tilde{x}^{j}$. Inserting (24), (26) and (27) into this equation gives

$\partial_{+-}^{2} \tilde{x}^{i}=-\tilde{H}_{j k}^{i} \partial_{+} x^{j} \partial_{-} x^{k}+i \tilde{H}_{j k}^{i} M_{m n}^{j}(0) \psi_{+}^{n} \psi_{+}^{m} \partial_{-} x^{k}-\frac{i}{2} \hat{\tilde{R}}_{j k l}^{i} \psi_{+}^{k} \psi_{+}^{l} \partial_{-} x^{j}+H . O$.

Likewise we can write a relation for $\partial_{+-}^{2} \tilde{x}^{i}$ using (26) or (27) as

$$
\partial_{+-}^{2} \tilde{x}^{i}=-M_{k j}^{i}(0) \partial_{+} x^{j} \partial_{-} x^{k}-\frac{i}{2} R_{j k l}^{i} \psi_{+}^{k} \psi_{+}^{l} \partial_{-} x^{j}+H . O .
$$

A simple comparison of (31) with (32) gives the following

$$
\begin{aligned}
\tilde{H}_{j k}^{i} & =M_{k j}^{i}(0) \\
-R_{j k l}^{i} & =-\hat{\tilde{R}}_{j k l}^{i}+2 \tilde{H}_{n j}^{i} \tilde{H}_{k l}^{n}
\end{aligned}
$$

We notice that (29) is the same as (33) , and $-\hat{\tilde{R}}_{j k l}^{i}+2 \tilde{H}_{n j}^{i} \tilde{H}_{k l}^{n}=-\tilde{R}_{j k l}^{i}$. Therefore we obtain $R_{j k l}^{i}=\tilde{R}_{j k l}^{i}$. We see that curvatures of the points $p$ and $\tilde{p}$ are constant and same. This implies that pseudoduality between two models based on Riemann normal coordinates must have same curvatures. We see from (29) and (30) that this transformation works in one way, and is not invertible in this special solution.

\subsubsection{General Solution}

Now we find the global solution to equation (23). We know that we can write $M_{k j}^{i}$ as the sum of symmetric and antisymmetric parts as follows

$$
M_{k j}^{i}=\frac{1}{2}\left(M_{k j}^{i}-M_{j k}^{i}\right)+\frac{1}{2}\left(M_{k j}^{i}+M_{j k}^{i}\right)
$$


Inserting antisymmetric part of this matrix into (23), and using the result (21) gives

$$
\partial_{n} M_{m j}^{i}=T_{k}^{i} R_{n j m}^{k}+2 M_{k j}^{i} \Gamma_{m n}^{k}-2 T_{l} \Gamma_{k j}^{l} \Gamma_{m n}^{k}
$$

If this equation is integrated, the result will be

$$
\begin{aligned}
M_{m j}^{i}= & M_{m j}^{i}(0)+2 M_{k j}^{i}(0) \int \Gamma_{m n}^{k} d x^{n}+4 M_{l j}^{i}(0) \int \Gamma_{m n}^{k} d x^{n} \int \Gamma_{k a}^{l} d x^{a} \\
& +\int T_{k}^{i}\left(R_{n j m}^{k}-2 \Gamma_{l j}^{k} \Gamma_{m n}^{l}\right) d x^{n}+\text { H.O. }
\end{aligned}
$$

and using $T_{m}^{i}=T_{m}^{i}(0)+\int M_{m j}^{i} d x^{j}$ we find $T$ up to the third order terms as follows

$$
\begin{aligned}
T_{m}^{i}= & T_{m}^{i}(0)+M_{m j}^{i}(0) x^{j}+2 M_{k j}^{i}(0) \int d x^{j} \int \Gamma_{m n}^{k} d x^{n} \\
& +4 M_{l j}^{i}(0) \int d x^{j} \int \Gamma_{m n}^{k} d x^{n} \int \Gamma_{k a}^{l} d x^{a}+T_{k}^{i}(0) \int d x^{j} \int\left(R_{n j m}^{k}-2 \Gamma_{l j}^{k} \Gamma_{m n}^{l}\right) d x^{n} \\
& +M_{k b}^{i}(0) \int d x^{j} \int\left(R_{n j m}^{k}-2 \Gamma_{l j}^{k} \Gamma_{m n}^{l}\right) x^{b} d x^{n}+\text { H.O. }
\end{aligned}
$$

which immediately leads to a final result for $M_{m j}^{i}$

$$
\begin{aligned}
M_{m j}^{i} & =M_{m j}^{i}(0)+2 M_{k j}^{i}(0) \int \Gamma_{m n}^{k} d x^{n}+4 M_{l j}^{i}(0) \int \Gamma_{m n}^{k} d x^{n} \int \Gamma_{k a}^{l} d x^{a} \\
& +T_{k}^{i}(0) \int\left(R_{n j m}^{k}-2 \Gamma_{l j}^{k} \Gamma_{m n}^{l}\right) d x^{n}+M_{k a}^{i}(0) \int\left(R_{n j m}^{k}-2 \Gamma_{l j}^{k} \Gamma_{m n}^{l}\right) x^{a} d x^{n}+\text { H.O. }
\end{aligned}
$$

One may find torsion and curvature relations using these explicit solutions as in the previous section. Let us inquire solutions by expressing equations (13) - (16) in terms of $T$ instead of finding explicit solutions.

If (21) is inserted in the pseudoduality equations (13)-(16) we get

$$
\begin{aligned}
\tilde{\psi}_{+}^{i} & =+T_{j}^{i} \psi_{+}^{j} \\
\partial_{-} \tilde{\psi}_{+}^{i} & =-T_{j}^{i} \partial_{-} \psi_{+}^{j}-M_{j m}^{i} \psi_{+}^{m} \partial_{-} x^{j} \\
\partial_{+} \tilde{x}^{i} & =+T_{j}^{i} \partial_{+} x^{j}-i M_{j m}^{i} \psi_{+}^{m} \psi_{+}^{j} \\
\partial_{-} \tilde{x}^{i} & =-T_{j}^{i} \partial_{-} x^{j}
\end{aligned}
$$

Using equations of motion for $\partial_{-} \tilde{\psi}_{+}^{i}$ and $\partial_{-} \psi_{+}^{j}$ in (36) , one finds

$$
\left(\tilde{H}_{m n}^{i}-\tilde{\Gamma}_{m n}^{i}\right) \tilde{\psi}_{+}^{m} \partial_{-} \tilde{x}^{n}=T_{j}^{i} \Gamma_{m n}^{j} \psi_{+}^{m} \partial_{-} x^{n}-M_{n m}^{i} \psi_{+}^{m} \partial_{-} x^{n}
$$


and inserting (35) and (38) into (39) leads to the following result

$$
\left(\tilde{H}_{m n}^{i}-\tilde{\Gamma}_{m n}^{i}\right) T_{a}^{m} T_{b}^{n}=M_{b a}^{i}-T_{j}^{i} \Gamma_{a b}^{j}
$$

Now taking $\partial_{-}$of (37) (or $\partial_{+}$of 38 ) leads to

$\partial_{+-}^{2} \tilde{x}^{i}=M_{j k}^{i} \partial_{+} x^{j} \partial_{-} x^{k}+T_{j}^{i} \partial_{+-}^{2} x^{j}-i \partial_{n} M_{j m}^{i} \psi_{+}^{m} \psi_{+}^{j} \partial_{-} x^{n}-i M_{j m}^{i} \partial_{-} \psi_{+}^{m} \psi_{+}^{j}-i M_{j m}^{i} \psi_{+}^{m} \partial_{-} \psi_{+}^{j}$

We use the equation of motion for $\partial_{+-}^{2} \tilde{x}^{i}, \partial_{+-}^{2} x^{j}$ and $\partial_{-} \psi_{+}^{m}$, and use the result (23) to get

$$
\begin{aligned}
\left(\tilde{H}_{j k}^{i}-\tilde{\Gamma}_{j k}^{i}\right) \partial_{+} \tilde{x}^{j} \partial_{-} \tilde{x}^{k}+\frac{i}{2} \hat{\tilde{R}}_{j k m}^{i} \tilde{\psi}_{+}^{k} \tilde{\psi}_{+}^{m} \partial_{-} \tilde{x}^{j}= & \left(M_{m n}^{i}-T_{j}^{i} \Gamma_{m n}^{j}\right) \partial_{+} x^{m} \partial_{-} x^{n} \\
& -\frac{i}{2} T_{j}^{i} R_{m n k}^{j} \psi_{+}^{n} \psi_{+}^{k} \partial_{-} x^{m}
\end{aligned}
$$

now using (35), (37) and (38) in (41) leads to

$$
\begin{aligned}
& -\left(\tilde{H}_{j k}^{i}-\tilde{\Gamma}_{j k}^{i}\right) T_{m}^{j} T_{n}^{k} \partial_{+} x^{m} \partial_{-} x^{n}+i\left(\tilde{H}_{j l}^{i}-\tilde{\Gamma}_{j l}^{i}\right) M_{k n}^{j} T_{m}^{l} \psi_{+}^{n} \psi_{+}^{k} \partial_{-} x^{m} \\
& -\frac{i}{2} \hat{\tilde{R}}_{a b c}^{i} T_{n}^{b} T_{k}^{c} T_{m}^{a} \psi_{+}^{n} \psi_{+}^{k} \partial_{-} x^{m}=\left(M_{m n}^{i}-T_{j}^{i} \Gamma_{m n}^{j}\right) \partial_{+} x^{m} \partial_{-} x^{n}-\frac{i}{2} T_{j}^{i} R_{m n k}^{j} \psi_{+}^{n} \psi_{+}^{k} \partial_{-} x^{m}
\end{aligned}
$$

which can be split into the following equations

$$
\begin{aligned}
& \left(\tilde{H}_{j k}^{i}-\tilde{\Gamma}_{j k}^{i}\right) T_{m}^{j} T_{n}^{k}=-M_{m n}^{i}+T_{j}^{i} \Gamma_{m n}^{j}=M_{[n m]}^{i} \\
& \frac{1}{2} T_{j}^{i} R_{m n k}^{j}=\frac{1}{2} \hat{\tilde{R}}_{a b c}^{i} T_{n}^{b} T_{k}^{c} T_{m}^{a}-\left(\tilde{H}_{j l}^{i}-\tilde{\Gamma}_{j l}^{i}\right) M_{[k n]}^{j} T_{m}^{l}
\end{aligned}
$$

we see that (40) and (42) are the same equations (by means of equation (21)). It is evident that right hand side of equation (42) is equal to the antisymmetric part of $M_{n m}^{i}$, and therefore, $\tilde{\Gamma}_{j k}^{i}=0$. Equation (43) can be written as

$$
\frac{1}{2} T_{j}^{i} R_{m n k}^{j}=\frac{1}{2}\left(\hat{\tilde{R}}_{a b c}^{i}-2 \tilde{H}_{j a}^{i} \tilde{H}_{b c}^{j}\right) T_{n}^{b} T_{k}^{c} T_{m}^{a}
$$

where we used (42). $\tilde{H}$ can be figured out by (42) using the initial values of $T$ and $M$, hence it is easy to see that $\tilde{H}_{m n}^{i}=M_{[n m]}^{i}(0)$. Therefore, we can write $\hat{\tilde{R}}_{a b c}^{i}-2 \tilde{H}_{j a}^{i} \tilde{H}_{b c}^{j}=\tilde{R}_{a b c}^{i}$, which leads to $R_{m n k}^{i}=\tilde{R}_{m n k}^{i}$ by equation (44). This means that curvatures will be related to each other by the relation $R_{m n k}^{i}=\tilde{R}_{m n k}^{i}$ around the point $p$ on $M$ where the transformation is identity, and $\tilde{R}_{m n k}^{i}$ is the curvature at point $\tilde{p}$. In this case all the points on manifold $M$ will be mapped to only one point $\tilde{p}$ on $\tilde{M}$ where riemann normal coordinates are used. 


\subsection{Orthonormal Coframes}

In this case we will present pseudoduality equations on the orthonormal coframe $S O(\mathbb{M})$. Equations of motion following from the action (6) in terms of the superfields are

$$
X_{+-}^{k}=X_{-+}^{k}=-\left[\Gamma_{i j}^{k}(X)-\mathcal{H}_{i j}^{k}(X)\right] X_{+}^{i} X_{-}^{j}
$$

where superfield $X$ has the form (5),$D_{+} X=X_{+}$and $\partial_{-} X=X_{-}$. We

choose an orthonormal frame $\left\{\Lambda^{i}\right\}$ with the riemannian connection $\Lambda_{j}^{i}$ on the superspace. If the superspace coordinates are defined by $z=\left(\sigma^{ \pm}, \theta^{+}\right)$, then one form is given by

$$
\Lambda^{i}=d z^{M} X_{M}^{i}
$$

Covariant derivatives of $X_{M}$ and $X_{M N}$ will be

$$
d X_{M}^{i}+\Lambda_{j}^{i} X_{M}^{j}=d z^{N} X_{M N}^{i}
$$

The Cartan structural equations are

$$
\begin{aligned}
d \Lambda^{i} & =-\Lambda_{j}^{i} \wedge \Lambda^{j} \\
d \Lambda_{j}^{i} & =-\Lambda_{k}^{i} \wedge \Lambda_{j}^{k}+\Omega_{j}^{i}
\end{aligned}
$$

where $\Omega_{j}^{i}=\frac{1}{2} \mathcal{R}_{j k l}^{i} \Lambda^{k} \wedge \Lambda^{l}$ is the curvature two form. Pseudoduality equations (11) and (12) are

$$
\tilde{X}_{ \pm}^{i}= \pm \mathcal{T}_{j}^{i} X_{ \pm}^{j}
$$

where $\mathcal{T}$ depends on superfield $X$. Taking the exterior derivative of both sides yields

$$
d \tilde{X}_{ \pm}^{i}= \pm d \mathcal{T}_{j}^{i} X_{ \pm}^{j} \pm \mathcal{T}_{j}^{i} d X_{ \pm}^{j}
$$

Inserting (47) in this equation gives

$$
-\tilde{\Lambda}_{j}^{i} \tilde{X}_{ \pm}^{j}+d z^{N} \tilde{X}_{ \pm N}^{i}= \pm d \mathcal{T}_{j}^{i} X_{ \pm}^{j} \mp \mathcal{T}_{j}^{i} \Lambda_{k}^{j} X_{ \pm}^{k} \pm d z^{N} \mathcal{T}_{j}^{i} X_{ \pm N}^{j}
$$

We now substitute (50) and arrange the terms to get

$$
d z^{N} \tilde{X}_{ \pm N}^{i}= \pm\left(d \mathcal{T}_{k}^{i}-\mathcal{T}_{j}^{i} \Lambda_{k}^{j}+\tilde{\Lambda}_{j}^{i} \mathcal{T}_{k}^{j}\right) X_{ \pm}^{k} \pm d z^{N} \mathcal{T}_{j}^{i} X_{ \pm N}^{j}
$$


We wedge the plus equation (upper sign) by $d z^{+}$and minus equation (lower sign) by $d z^{-}$, and find the following equations

$$
\begin{gathered}
d z^{+} \wedge d z^{-} \tilde{X}_{+-}^{i}=d z^{+} \wedge\left(d \mathcal{T}_{k}^{i}-\mathcal{T}_{j}^{i} \Lambda_{k}^{j}+\tilde{\Lambda}_{j}^{i} \mathcal{T}_{k}^{j}\right) X_{+}^{k}+d z^{+} \wedge d z^{-} \mathcal{T}_{j}^{i} X_{+-}^{j} \\
d z^{-} \wedge d z^{+} \tilde{X}_{-+}^{i}=-d z^{-} \wedge\left(d \mathcal{T}_{k}^{i}-\mathcal{T}_{j}^{i} \Lambda_{k}^{j}+\tilde{\Lambda}_{j}^{i} \mathcal{T}_{k}^{j}\right) X_{-}^{k}-d z^{-} \wedge d z^{+} \mathcal{T}_{j}^{i} X_{-+}^{j}
\end{gathered}
$$

Since $X_{+-}=X_{-+}$(also with tilde) and $d z^{+} \wedge d z^{-}=d z^{-} \wedge d z^{+}$we may find the constraint relations by equating left hand sides

$$
\begin{array}{r}
2 d z^{+} \wedge d z^{-} \mathcal{T}_{k}^{i} X_{+-}^{k}+d z^{+} \wedge\left(d \mathcal{T}_{k}^{i}-\mathcal{T}_{j}^{i} \Lambda_{k}^{j}+\tilde{\Lambda}_{j}^{i} \mathcal{T}_{k}^{j}\right) X_{+}^{k} \\
+d z^{-} \wedge\left(d \mathcal{T}_{k}^{i}-\mathcal{T}_{j}^{i} \Lambda_{k}^{j}+\tilde{\Lambda}_{j}^{i} \mathcal{T}_{k}^{j}\right) X_{-}^{k}=0
\end{array}
$$

we substitute the equations of motion (45)

$$
\begin{aligned}
& -2 d z^{+} \wedge d z^{-} \mathcal{T}_{k}^{i}\left[\Gamma_{m n}^{k}-\mathcal{H}_{m n}^{k}\right] X_{+}^{m} X_{-}^{n}+d z^{+} \wedge\left(d \mathcal{T}_{k}^{i}-\mathcal{T}_{j}^{i} \Lambda_{k}^{j}+\tilde{\Lambda}_{j}^{i} \mathcal{T}_{k}^{j}\right) X_{+}^{k} \\
& +d z^{-} \wedge\left(d \mathcal{T}_{k}^{i}-\mathcal{T}_{j}^{i} \Lambda_{k}^{j}+\tilde{\Lambda}_{j}^{i} \mathcal{T}_{k}^{j}\right) X_{-}^{k}=0
\end{aligned}
$$

and we use $d z^{ \pm} X_{ \pm}^{n}=\Lambda^{n}-d z^{\mp} X_{\mp}^{n}$ to get

$$
\begin{aligned}
& -d z^{+} \wedge \mathcal{T}_{k}^{i}\left(\Gamma_{m n}^{k}-\mathcal{H}_{m n}^{k}\right) X_{+}^{m} \Lambda^{n}-d z^{-} \wedge \mathcal{T}_{k}^{i}\left(\Gamma_{m n}^{k}+\mathcal{H}_{m n}^{k}\right) X_{-}^{m} \Lambda^{n} \\
& +d z^{+} \wedge\left(d \mathcal{T}_{k}^{i}-\mathcal{T}_{j}^{i} \Lambda_{k}^{j}+\tilde{\Lambda}_{j}^{i} \mathcal{T}_{k}^{j}\right) X_{+}^{k}+d z^{-} \wedge\left(d \mathcal{T}_{k}^{i}-\mathcal{T}_{j}^{i} \Lambda_{k}^{j}+\tilde{\Lambda}_{j}^{i} \mathcal{T}_{k}^{j}\right) X_{-}^{k}=0
\end{aligned}
$$

Now we define the following tensors

$$
\begin{aligned}
& d z^{-} \mathcal{U}_{k-}^{i}=\left(d \mathcal{T}_{k}^{i}-\mathcal{T}_{j}^{i} \Lambda_{k}^{j}+\tilde{\Lambda}_{j}^{i} \mathcal{T}_{k}^{j}\right)-\mathcal{T}_{j}^{i}\left(\Gamma_{k n}^{j}-\mathcal{H}_{k n}^{j}\right) \Lambda^{n} \\
& d z^{+} \mathcal{U}_{k+}^{i}=-\left(d \mathcal{T}_{k}^{i}-\mathcal{T}_{j}^{i} \Lambda_{k}^{j}+\tilde{\Lambda}_{j}^{i} \mathcal{T}_{k}^{j}\right)+\mathcal{T}_{j}^{i}\left(\Gamma_{k n}^{j}+\mathcal{H}_{k n}^{j}\right) \Lambda^{n}
\end{aligned}
$$

which satisfies the equation (55)

$$
d z^{+} \wedge d z^{-} \mathcal{U}_{k-}^{i} X_{+}^{k}-d z^{-} \wedge d z^{+} \mathcal{U}_{k+}^{i} X_{-}^{k}=0
$$

They also yield the result

$$
d z^{-} \mathcal{U}_{k-}^{i}+d z^{+} \mathcal{U}_{k+}^{i}=2 \mathcal{T}_{j}^{i} \mathcal{H}_{k n}^{j} \Lambda^{n}
$$

which gives

$$
\begin{aligned}
& d z^{+} \wedge d z^{-} \mathcal{U}_{k-}^{i}=2 d z^{+} \wedge \mathcal{T}_{j}^{i} \mathcal{H}_{k n}^{j} \Lambda^{n} \\
& d z^{-} \wedge d z^{+} \mathcal{U}_{k+}^{i}=2 d z^{-} \wedge \mathcal{T}_{j}^{i} \mathcal{H}_{k n}^{j} \Lambda^{n}
\end{aligned}
$$


If these equations are substituted into (58), one obtains

$$
2 d z^{+} \wedge \mathcal{T}_{j}^{i} \mathcal{H}_{k n}^{j} \Lambda^{n} X_{+}^{k}-2 d z^{-} \wedge \mathcal{T}_{j}^{i} \mathcal{H}_{k n}^{j} \Lambda^{n} X_{-}^{k}=0
$$

and using (46) gives the final result

$$
2 d z^{+} \wedge d z^{-} \mathcal{T}_{j}^{i} \mathcal{H}_{k n}^{j} X_{-}^{n} X_{+}^{k}-2 d z^{-} \wedge d z^{+} \mathcal{T}_{j}^{i} \mathcal{H}_{k n}^{j} X_{+}^{n} X_{-}^{k}=0
$$

which shows that

$$
d z^{+} \wedge d z^{-} \mathcal{T}_{j}^{i} \mathcal{H}_{k n}^{j} X_{+}^{k} X_{-}^{n}=0
$$

Therefore, we conclude that $\mathcal{H}=0$, and $\mathcal{U}_{k-}^{i}=\mathcal{U}_{k+}^{i}=0$ by equations (60) and (61). Finally equation (56) and (57) gives the following result

$$
\left(d \mathcal{T}_{k}^{i}-\mathcal{T}_{j}^{i} \Lambda_{k}^{j}+\tilde{\Lambda}_{j}^{i} \mathcal{T}_{k}^{j}\right)=\mathcal{T}_{j}^{i} \Gamma_{k n}^{j} \Lambda^{n}
$$

If we insert the equations of motion into (51) and (52), we obtain

$$
\begin{array}{r}
-d z^{+} \wedge d z^{-}\left(\tilde{\boldsymbol{\Gamma}}_{j k}^{i}-\tilde{\mathcal{H}}_{j k}^{i}\right) \tilde{X}_{+}^{j} \tilde{X}_{-}^{k}=d z^{+} \wedge\left(d \mathcal{T}_{k}^{i}-\mathcal{T}_{j}^{i} \Lambda_{k}^{j}+\tilde{\Lambda}_{j}^{i} \mathcal{T}_{k}^{j}\right) X_{+}^{k} \\
-d z^{+} \wedge d z^{-} \mathcal{T}_{j}^{i} \boldsymbol{\Gamma}_{m n}^{i} X_{+}^{m} X_{-}^{n} \\
-d z^{-} \wedge d z^{+}\left(\tilde{\boldsymbol{\Gamma}}_{j k}^{i}-\tilde{\mathcal{H}}_{j k}^{i}\right) \tilde{X}_{+}^{j} \tilde{X}_{-}^{k}=-d z^{-} \wedge\left(d \mathcal{T}_{k}^{i}-\mathcal{T}_{j}^{i} \Lambda_{k}^{j}+\tilde{\Lambda}_{j}^{i} \mathcal{T}_{k}^{j}\right) X_{-}^{k} \\
+d z^{-} \wedge d z^{+} \mathcal{T}_{j}^{i} \Gamma_{m n}^{i} X_{+}^{m} X_{-}^{n}
\end{array}
$$

Inserting $d z^{-} X_{-}^{k}=\Lambda-d z^{+} X_{+}$(also with tilde) and $\tilde{X}_{+}$(50) into (66), and $d z^{+} X_{+}=\Lambda-d z^{-} X_{-}$(also with tilde) and $\tilde{X}_{-}$(50) into (67) gives

$$
\begin{gathered}
-d z^{+} \wedge\left(\tilde{\boldsymbol{\Gamma}}_{m n}^{i}-\tilde{\mathcal{H}}_{m n}^{i}\right) T_{k}^{m} \tilde{\Lambda}^{n}=d z^{+} \wedge\left(d \mathcal{T}_{k}^{i}-\mathcal{T}_{j}^{i} \Lambda_{k}^{j}+\tilde{\Lambda}_{j}^{i} \mathcal{T}_{k}^{j}\right)-d z^{+} \wedge \mathcal{T}_{j}^{i} \Gamma_{k n}^{i} \Lambda^{n} \\
-d z^{-} \wedge\left(\tilde{\boldsymbol{\Gamma}}_{m n}^{i}+\tilde{\mathcal{H}}_{m n}^{i}\right) T_{k}^{m} \tilde{\Lambda}^{n}=-d z^{-} \wedge\left(d \mathcal{T}_{k}^{i}-\mathcal{T}_{j}^{i} \Lambda_{k}^{j}+\tilde{\Lambda}_{j}^{i} \mathcal{T}_{k}^{j}\right)+d z^{-} \wedge \mathcal{T}_{j}^{i} \Gamma_{k n}^{i} \Lambda^{n}
\end{gathered}
$$

where we cancelled out $X_{+}^{k}$ in (68) and $X_{-}^{k}$ in (69). We notice that right-hand sides of these equations become zero by means of the constraint relation (65), and we are left with

$$
\begin{aligned}
& \left(\tilde{\boldsymbol{\Gamma}}_{m n}^{i}-\tilde{\mathcal{H}}_{m n}^{i}\right) \mathcal{T}_{k}^{m} \tilde{\Lambda}^{n}=0 \\
& \left(\tilde{\boldsymbol{\Gamma}}_{m n}^{i}+\tilde{\mathcal{H}}_{m n}^{i}\right) \mathcal{T}_{k}^{m} \tilde{\Lambda}^{n}=0
\end{aligned}
$$

This shows that on the transformed superspace we must have $\tilde{\boldsymbol{\Gamma}}=0$ and $\tilde{\mathcal{H}}_{m n}^{i}=0$. We may find the relation between curvatures of the spaces using 
(65). We may define the connection one form $\Lambda_{k}^{j}=\Gamma_{k n}^{j} \Lambda^{n}$, and hence (65) is reduced to

$$
\left(d \mathcal{T}_{k}^{i}-2 \mathcal{T}_{j}^{i} \Lambda_{k}^{j}+\tilde{\Lambda}_{j}^{i} \mathcal{T}_{k}^{j}\right)=0
$$

Taking exterior derivative, and using again (72) together with (49) gives

$$
\mathcal{T}_{j}^{i} \Omega_{k}^{j}=\tilde{\Omega}_{j}^{i} \mathcal{T}_{k}^{j}
$$

where new orthonormal coframe is replaced by $2 \Lambda$ with the same curvature two form $\Omega$ on the manifold $\mathbb{M}$. It is obvious that integrability condition of this equation followed by the use of (46) and (50) yields a curvature relation between two $(1,0)$ supersymmetric sigma models which tied together with pseudoduality, which can be reduced to the same results found in the previous section. The reason why we get a positive sign in curvature expression in component expansion method is because of anticommuting grassmann numbers. This gives that pseudoduality transformation can be performed only if two sigma models are based on symmetric spaces with opposite curvatures on target spaces $\mathbb{M}$ and $\tilde{\mathbb{M}}$.

\section{Pseudoduality in $(1,1)$ Supersymmetric Sigma Models}

In this case [1] the classical spacetime $\Sigma$ can be enlarged to the superspace $\Xi^{1,1}$ by adding Grassmann coordinates of opposite chirality. We will have

one left-handed supercharge $Q_{+}$, and one right-handed supercharge $Q_{-}$as given by (2). The supersymmetry algebra can be written as

$$
\left\{Q_{ \pm}, Q_{ \pm}\right\}=2 i P_{ \pm} \quad\left\{Q_{+}, Q_{-}\right\}=0
$$

where $P_{ \pm}=-\partial_{ \pm}$. The supersymmetry transformations will be

$$
\begin{aligned}
\delta_{\epsilon} x^{i} & =\epsilon^{+} \psi_{+}^{i}+\epsilon^{-} \psi_{-}^{i} \\
\delta_{\epsilon} \psi_{+}^{i} & =i \epsilon^{+} \partial_{+} x^{i}+\epsilon^{-}\left(\Gamma_{j k}^{i}+H_{j k}^{i}\right) \psi_{+}^{i} \psi_{-}^{k} \\
\delta_{\epsilon} \psi_{-}^{i} & =-\epsilon^{+}\left(\Gamma_{j k}^{i}+H_{j k}^{i}\right) \psi_{+}^{j} \psi_{-}^{k}+i \epsilon^{-} \partial_{-} x^{i}
\end{aligned}
$$

where $\epsilon^{ \pm}$is the constant anticommuting parameter. 


\subsection{Components}

The superfield is written as

$$
X=x+\theta^{+} \psi_{+}+\theta^{-} \psi_{-}+\theta^{+} \theta^{-} F
$$

where $X: \Xi^{1,1} \rightarrow M$. We define the $(1,1)$ superspace $\Xi^{1,1}=\left(\sigma^{+}, \sigma^{-}, \theta^{+}, \theta^{-}\right)$, where $\left(\sigma^{+}, \sigma^{-}\right)$are the null coordinates, and $\left(\theta^{+}, \theta^{-}\right)$are the Grassman coordinates of opposite chirality. The action of the theory is

$$
S=\int d^{2} \sigma d^{2} \theta\left(G_{i j}+B_{i j}\right) D_{+} X^{i} D_{-} X^{j}
$$

where supercovariant derivatives are given in (1). Similar definitions can be written for pseudodual model with tilde. First order expansion of $G_{i j}$ and $B_{i j}$, followed by the $d^{2} \theta$ integral gives

$S=-\int d^{2} x\left[\left(g_{i j}+b_{i j}\right) \partial_{+} x^{i} \partial_{-} x^{j}+i g_{i j} \psi_{+}^{i} \nabla_{-}^{(-)} \psi_{+}^{j}+i g_{i j} \psi_{-}^{i} \nabla_{+}^{(+)} \psi_{-}^{j}-\frac{1}{2} \hat{R}_{b n a m}^{+} \psi_{+}^{m} \psi_{-}^{n} \psi_{+}^{a} \psi_{-}^{b}\right]$

where $\nabla_{ \pm}^{( \pm)} \psi_{\mp}^{j}=\nabla_{ \pm} \psi_{\mp}^{j} \pm H_{m n}^{j} \psi_{\mp}^{m} \partial_{ \pm} x^{n}$, and $\hat{R}_{\text {bnam }}^{ \pm}=R_{\text {bnam }} \pm D_{a} H_{n m b} \mp$ $D_{m} H_{n a b}+H_{b a j} H_{m n}^{j}-H_{n a j} H_{m b}^{j}$.

Equations of motion following from this action will be

$$
\begin{aligned}
F^{i}= & \left(\Gamma_{j k}^{i}-H_{j k}^{i}\right) \psi_{+}^{j} \psi_{-}^{k} \\
\nabla_{-}^{(-)} \psi_{+}^{i}= & \frac{i}{2}\left(\hat{R}^{+}\right)_{j m n}^{i} \psi_{-}^{n} \psi_{+}^{j} \psi_{-}^{m} \\
\nabla_{+}^{(+)} \psi_{-}^{i}= & \frac{i}{2}\left(\hat{R}^{+}\right)_{j m n}^{i} \psi_{+}^{n} \psi_{-}^{j} \psi_{+}^{m} \\
\square x^{k}= & i\left(\hat{R}^{-}\right)_{n i m}^{k} \psi_{+}^{i} \psi_{+}^{m} \partial_{-} x^{n}+i\left(\hat{R}^{+}\right)_{n i m}^{k} \psi_{-}^{i} \psi_{-}^{m} \partial_{+} x^{n} \\
& -\left(\hat{D}^{k} \hat{R}_{\text {bnam }}^{+}\right) \psi_{+}^{m} \psi_{-}^{n} \psi_{+}^{a} \psi_{-}^{b}
\end{aligned}
$$

where $\hat{D}^{k} \hat{R}_{\text {bnam }}^{+}=D^{k} \hat{R}_{\text {bnam }}^{+}+H_{j n}^{k}\left(\hat{R}^{+}\right)_{\text {bam }}^{j}-H_{j b}^{k}\left(\hat{R}^{+}\right)_{n a m}^{j}+H_{j a}^{k}\left(\hat{R}^{+}\right)_{m b n}^{j}-$ $H_{j m}^{k}\left(\hat{R}^{+}\right)_{a b n}^{j}$.

Pseudoduality transformations are

$$
\begin{aligned}
& D_{+} \tilde{X}^{i}=+\mathcal{T}_{j}^{i} D_{+} X^{j} \\
& D_{-} \tilde{X}^{i}=-\mathcal{T}_{j}^{i} D_{-} X^{j}
\end{aligned}
$$


where $\mathcal{T}$ is a function of superfield (74). Transformation matrix $\mathcal{T}$ can be expanded as $\mathcal{T}(X)=T(x)+\theta^{+} \psi_{+}^{k} \partial_{k} T(x)+\theta^{-} \psi_{-}^{k} \partial_{k} T(x)+\theta^{+} \theta^{-} F^{k} \partial_{k} T(x)-$ $\theta^{+} \theta^{-} \psi_{+}^{k} \psi_{-}^{l} \partial_{k} \partial_{l} T(x)$. If pseudoduality transformations are written in components, first equation (80) yields the following set of equations

$$
\begin{aligned}
\tilde{\psi}_{+}^{i} & =T_{j}^{i} \psi_{+}^{j} \\
\tilde{F}^{i} & =T_{j}^{i} F^{j}-M_{j k}^{i} \psi_{+}^{j} \psi_{-}^{k} \\
\partial_{+} \tilde{x}^{i} & =T_{j}^{i} \partial_{+} x^{j}+i M_{j k}^{i} \psi_{+}^{j} \psi_{+}^{k} \\
\partial_{+} \tilde{\psi}_{-}^{i} & =T_{j}^{i} \partial_{+} \psi_{-}^{j}-2 i M_{[j k]}^{i} \psi_{+}^{j} F^{k}+M_{k j}^{i} \psi_{-}^{j} \partial_{+} x^{k}+i \partial_{l} M_{[j k]}^{i} \psi_{+}^{k} \psi_{-}^{l} \psi_{+}^{j}
\end{aligned}
$$

where $M_{j k}^{i}=\partial_{k} T_{j}^{i}$, and $M_{[j k]}^{i}$ represents the antisymmetric part of $M_{j k}^{i}$. Second equation (81) will produce

$$
\begin{aligned}
\tilde{\psi}_{-}^{i} & =-T_{j}^{i} \psi_{-}^{j} \\
\tilde{F}^{i} & =-T_{j}^{i} F^{j}+M_{k j}^{i} \psi_{+}^{j} \psi_{-}^{k} \\
\partial_{-} \tilde{x}^{i} & =-T_{j}^{i} \partial_{-} x^{j}-i M_{j k}^{i} \psi_{-}^{j} \psi_{-}^{k} \\
\partial_{-} \tilde{\psi}_{+}^{i} & =-T_{j}^{i} \partial_{-} \psi_{+}^{j}-2 i M_{[j k]}^{i} \psi_{-}^{j} F^{k}-M_{k j}^{i} \psi_{+}^{j} \partial_{-} x^{k}-i \partial_{l} M_{[j k]}^{i} \psi_{-}^{k} \psi_{+}^{l} \psi_{-}^{j}
\end{aligned}
$$

We can find constraint relations using these equations. If (83) is set equal to (87), and equation of motion (76) is used, the result follows

$$
T_{j}^{i}\left(\Gamma_{m n}^{i}-H_{m n}^{j}\right)=M_{(m n)}^{i}
$$

where $M_{(m n)}^{i}$ is the symmetric part of $M_{m n}^{i}$. We immediately notice that $H_{m n}^{j}=0$, and we are left with

$$
T_{j}^{i} \Gamma_{m n}^{j}=M_{(m n)}^{i}
$$

We next take $\partial_{-}$of (82) and set equal to (89) followed by the equations of motion (176) and (77) to obtain

$$
\left[2 M_{(m n)}^{i}-2 T_{j}^{i} \Gamma_{m n}^{j}\right] \psi_{+}^{m} \partial_{-} x^{n}=-i\left[\partial_{a} M_{[b c]}^{i}+2 M_{[c k]}^{i} \Gamma_{a b}^{k}+T_{j}^{i} R_{a b c}^{j}\right] \psi_{-}^{c} \psi_{+}^{a} \psi_{-}^{b}
$$

Real part of this equation is simply (91), and complex part will produce

$$
\partial_{a} M_{[b c]}^{i}+2 M_{[c k]}^{i} \Gamma_{a b}^{k}+T_{j}^{i} R_{a b c}^{j}=0
$$


We now take $\partial_{+}$of $(86)$ and set equal to (85) followed by the equations of motion (76) and (78) to get

$$
\left[2 M_{(m n)}^{i}-2 T_{j}^{i} \Gamma_{m n}^{j}\right] \psi_{-}^{m} \partial_{+} x^{n}=-i\left[\partial_{a} M_{[b c]}^{i}+2 M_{[c k]}^{i} \Gamma_{a b}^{k}+T_{j}^{i} R_{a b c}^{j}\right] \psi_{+}^{c} \psi_{-}^{a} \psi_{+}^{b}
$$

This equation is similar to (92), and we again notice that real part of this equation is equal to (91), and complex part is (93). We finally take $\partial_{-}$of (84), $\partial_{+}$of (88), and set them equal to each other to find out the remaining constraints

$$
\begin{aligned}
2 M_{(j k)}^{i} \partial_{+} x^{j} \partial_{-} x^{k} & +2 T_{j}^{i} \partial_{+-}^{2} x^{j}=2 i M_{[k j]}^{i} \psi_{+}^{j} \partial_{-} \psi_{+}^{k}+i \partial_{n} M_{[k j]} \psi_{+}^{j} \psi_{+}^{k} \partial_{-} x^{n} \\
& +2 i M_{[k j]}^{i} \psi_{-}^{j} \partial_{+} \psi_{-}^{k}+i \partial_{n} M_{[k j]} \psi_{-}^{j} \psi_{-}^{k} \partial_{+} x^{n}
\end{aligned}
$$

using equations of motion for $\partial_{+-}^{2} x^{j}(\overline{79}), \partial_{-} \psi_{+}^{j}(77)$ and $\partial_{+} \psi_{-}^{j}(\mathbf{7 8})$ yields

$$
\begin{aligned}
& \left(2 M_{(m n)}^{i}-2 T_{j}^{i} \Gamma_{m n}^{j}\right) \partial_{+} x^{m} \partial_{-} x^{n}+i\left(T_{j}^{i} R_{a b c}^{j}+2 M_{[k b]}^{i} \Gamma_{c a}^{k}+\partial_{a} M_{[b c]}^{i}\right) \psi_{+}^{b} \psi_{+}^{c} \partial_{-} x^{a} \\
& +i\left(T_{j}^{i} R_{a b c}^{j}+2 M_{[k b]}^{i} \Gamma_{c a}^{k}+\partial_{a} M_{[b c]}^{i}\right) \psi_{-}^{b} \psi_{-}^{c} \partial_{+} x^{a} \\
& -\left(T_{j}^{i} D^{j} R_{a b c d}+M_{[d k]}^{i} R_{c a b}^{k}+M_{[b k]}^{i} R_{a c d}^{k}\right) \psi_{+}^{d} \psi_{-}^{b} \psi_{+}^{c} \psi_{-}^{a}=0
\end{aligned}
$$

If this equation is split into real and complex parts the following results are obtained

$$
\begin{gathered}
\left(2 M_{(m n)}^{i}-2 T_{j}^{i} \Gamma_{m n}^{j}\right) \partial_{+} x^{m} \partial_{-} x^{n}=\left(T_{j}^{i} D^{j} R_{a b c d}+M_{[d k]}^{i} R_{c a b}^{k}+M_{[b k]}^{i} R_{a c d}^{k}\right) \psi_{+}^{d} \psi_{-}^{b} \psi_{+}^{c} \psi_{-}^{a} \\
\left(T_{j}^{i} R_{a b c}^{j}+2 M_{[k b]}^{i} \Gamma_{c a}^{k}+\partial_{a} M_{[b c]}^{i}\right) \psi_{+}^{b} \psi_{+}^{c} \partial_{-} x^{a} \\
+\left(T_{j}^{i} R_{a b c}^{j}+2 M_{[k b]}^{i} \Gamma_{c a}^{k}+\partial_{a} M_{[b c]}^{i}\right) \psi_{-}^{b} \psi_{-}^{c} \partial_{+} x^{a}=0
\end{gathered}
$$

First equation leads to the following results

$$
\begin{gathered}
M_{(m n)}^{i}=T_{j}^{i} \Gamma_{m n}^{j} \\
T_{j}^{i} D^{j} R_{a b c d}+M_{[d k]}^{i} R_{c a b}^{k}+M_{[b k]}^{i} R_{a c d}^{k}=0
\end{gathered}
$$

where (97) is the same as (91). Second equation gives

$$
T_{j}^{i} R_{a b c}^{j}+2 M_{[k b]}^{i} \Gamma_{c a}^{k}+\partial_{a} M_{[b c]}^{i}=0
$$

which is the same equation as (93) with $b \leftrightarrow c$. Obviously we have three independent constraint relations, which are (91), (93), and (98). 
Now we can find out pseudodual fields, and relations between two sigma models based on $M$ and $\tilde{M}$ by means of pseudoduality equations. Using (83) or (87), and equation of motion (76) for $F^{j}$ we get

$$
\tilde{F}^{i}=M_{[n m]}^{i} \psi_{+}^{m} \psi_{-}^{n}
$$

Also definition of $\tilde{F}^{i}$ gives that

$$
\begin{aligned}
\tilde{F}^{i} & =\left(\tilde{\Gamma}_{j k}^{i}-\tilde{H}_{j k}^{i}\right) \tilde{\psi}_{+}^{j} \tilde{\psi}_{-}^{k} \\
& =-\left(\tilde{\Gamma}_{j k}^{i}-\tilde{H}_{j k}^{i}\right) T_{m}^{j} T_{n}^{k} \psi_{+}^{m} \psi_{-}^{n}
\end{aligned}
$$

where we used (82) and (86). Comparison of (100) with (101) gives that

$$
\left(\tilde{\Gamma}_{j k}^{i}-\tilde{H}_{j k}^{i}\right) T_{m}^{j} T_{n}^{k}=M_{[m n]}^{i}
$$

Hence we obtain that $\tilde{\Gamma}_{j k}^{i}=0$. This means that pseudoduality transformation will be from any point on $M$ to only one point where $\tilde{\Gamma}$ vanishes on $\tilde{M}$. We know that this is consistent with Riemann normal coordinates. We are left with

$$
\tilde{H}_{j k}^{i} T_{m}^{j} T_{n}^{k}=M_{[n m]}^{i}
$$

We next consider (85). Using equations of motion (76) and (78) we obtain

$$
\partial_{+} \tilde{\psi}_{-}^{i}=M_{[m n]}^{i} \psi_{-}^{m} \partial_{+} x^{n}-\frac{i}{2} T_{j}^{i} R_{a b c}^{j} \psi_{+}^{c} \psi_{-}^{a} \psi_{+}^{b}
$$

where we used the constraint (93). On the other hand we can write the equation of motion (78) on $\tilde{M}$ as

$$
\begin{aligned}
\partial_{+} \tilde{\psi}_{-}^{i} & =-\tilde{H}_{j k}^{i} \tilde{\psi}_{-}^{j} \partial_{+} \tilde{x}^{k}+\frac{i}{2}\left(\hat{\tilde{R}}^{+}\right)_{j m n}^{i} \tilde{\psi}_{+}^{n} \tilde{\psi}_{-}^{j} \tilde{\psi}_{+}^{m} \\
& =\tilde{H}_{j k}^{i} T_{m}^{j} T_{n}^{k} \psi_{-}^{m} \partial_{+} x^{n}+i\left(\tilde{H}_{j k}^{i} T_{a}^{j} M_{[b c]}^{k}-\frac{1}{2}\left(\hat{\tilde{R}}^{+}\right)_{j m n}^{i} T_{c}^{n} T_{a}^{j} T_{b}^{m}\right) \psi_{+}^{c} \psi_{-}^{a} \psi_{+}^{b}
\end{aligned}
$$

where we used (82), (84) and (86) in the first line of (105). If we compare (104) with (105) we see that

$$
\begin{aligned}
\tilde{H}_{j k}^{i} T_{m}^{j} T_{n}^{k} & =M_{[m n]}^{i} \\
\frac{1}{2} T_{j}^{i} R_{a b c}^{j} & =\frac{1}{2}\left(\hat{\tilde{R}}^{+}\right)_{j m n}^{i} T_{c}^{n} T_{a}^{j} T_{b}^{m}-\tilde{H}_{j k}^{i} T_{a}^{j} M_{[b c]}^{k}
\end{aligned}
$$


From (103) and (106) it is obvious that antisymmetric part of $M_{m n}^{i}$ disappears, $M_{[m n]}^{i}=0$, which leads to the result $\tilde{H}_{j k}^{i}=0$. Hence (107) is reduced to

$$
T_{j}^{i} R_{a b c}^{j}=\tilde{R}_{j m n}^{i} T_{c}^{n} T_{a}^{j} T_{b}^{m}
$$

We now simplify right hand side of (89)). We use equations of motion (76) and (77) and arrange the terms to get

$$
\partial_{-} \tilde{\psi}_{+}^{i}=\frac{i}{2} T_{j}^{i} R_{a b c}^{j} \psi_{-}^{c} \psi_{+}^{a} \psi_{-}^{b}
$$

where we used the constraint (93). Also equation of motion for $\partial_{-} \tilde{\psi}_{+}^{i}$ on $\tilde{M}$ gives

$$
\begin{aligned}
\partial_{-} \tilde{\psi}_{+}^{i} & =\frac{i}{2} \tilde{R}_{j m n}^{i} \tilde{\psi}_{-}^{n} \tilde{\psi}_{+}^{j} \tilde{\psi}_{-}^{m} \\
& =\frac{i}{2} \tilde{R}_{j m n}^{i} T_{a}^{n} T_{b}^{j} T_{c}^{m} \psi_{-}^{a} \psi_{+}^{b} \psi_{-}^{c}
\end{aligned}
$$

A comparison of (109) with (110) gives (108). When we take $\partial_{-}$of 84 , and using relevant equations of motion together with the constraints (93) and (98) gives

$$
\partial_{+-}^{2} \tilde{x}^{i}=\frac{i}{2} T_{j}^{i} R_{a b c}^{j} \psi_{-}^{b} \psi_{-}^{c} \partial_{+} x^{a}-\frac{i}{2} T_{j}^{i} R_{a b c}^{j} \psi_{+}^{b} \psi_{+}^{c} \partial_{-} x^{a}
$$

Likewise on $\tilde{M}$ we obtain

$$
\begin{aligned}
\partial_{+-}^{2} \tilde{x}^{i}= & \frac{i}{2} \tilde{R}_{a b c}^{i} \tilde{\psi}_{+}^{b} \tilde{\psi}_{+}^{c} \partial_{-} \tilde{x}^{a}+\frac{i}{2} \tilde{R}_{a b c}^{i} \tilde{\psi}_{-}^{b} \tilde{\psi}_{-}^{c} \partial_{+} \tilde{x}^{a}-\frac{1}{2} \tilde{D}^{i} \tilde{R}_{a b c d} \tilde{\psi}_{+}^{d} \tilde{\psi}_{-}^{b} \tilde{\psi}_{+}^{c} \tilde{\psi}_{-}^{a} \\
= & -\frac{i}{2} \tilde{R}_{m n k}^{i} T_{b}^{n} T_{c}^{k} T_{a}^{m} \psi_{+}^{b} \psi_{+}^{c} \partial_{-} x^{a}+\frac{i}{2} \tilde{R}_{m n k}^{i} T_{b}^{n} T_{c}^{k} T_{a}^{m} \psi_{-}^{b} \psi_{-}^{c} \partial_{+} x^{a} \\
& -\frac{1}{2} \tilde{D}^{i} \tilde{R}_{j k m n} T_{d}^{n} T_{b}^{k} T_{c}^{m} T_{a}^{j} \psi_{+}^{d} \psi_{-}^{b} \psi_{+}^{c} \psi_{-}^{a}
\end{aligned}
$$

A quick comparison shows that we obtain equation (108), and $\tilde{D}^{i} \tilde{R}_{j k m n}=0$. We notice that covariant derivatives of curvatures on both spaces vanish while curvatures are constants, and related to each other by (108). This obeys that both models are based on symmetric spaces. 


\subsection{Orthonormal Coframes}

Equations of motion following from (75) are

$$
X_{-+}^{k}=-\left[\Gamma_{i j}^{k}(X)-\mathcal{H}_{i j}^{k}(X)\right] X_{+}^{i} X_{-}^{j}
$$

where $X_{+}=D_{+} X, X_{-}=D_{-} X$ and $X_{-+}=D_{+} D_{-} X$. On the contrary to (1, $0)$ case, this time one writes that $X_{-+}=-X_{+-}$and $\left\{X_{+}, X_{-}\right\}=0$, where $\{$,$\} defines the anticommutation. Superspace coordinates are z=\left(\sigma^{ \pm}, \theta^{ \pm}\right)$, and orthonormal frame can be chosen as $\left\{\Lambda^{i}\right\}$ with connection one form $\left\{\Lambda_{j}^{i}\right\}$. Similar to (46) and (47) one form $\left\{\Lambda^{i}\right\}$ and covariant derivative of $X_{M}$ can be written as

$$
\begin{aligned}
\Lambda^{i} & =d z^{M} X_{M}^{i} \\
d X_{M}^{i}+\Lambda_{j}^{i} X_{M}^{j} & =d z^{N} X_{M N}^{i}
\end{aligned}
$$

Pseudoduality relations are

$$
\tilde{X}_{ \pm}^{i}= \pm \mathcal{T}_{j}^{i} X_{ \pm}^{j}
$$

We are going to mimic the calculations performed in $(1,0)$ case except notable differences $d z^{+} \wedge d z^{-}=-d z^{-} \wedge d z^{+}, X_{+-}=-X_{-+}$, and $X_{+} X_{-}=-X_{-} X_{+}$. We take exterior derivative of (116), and then use (115) for both manifolds, and arrange the terms to get

$$
d z^{N} \tilde{X}_{ \pm N}^{i}= \pm\left(d \mathcal{T}_{k}^{i}-\mathcal{T}_{j}^{i} \Lambda_{k}^{j}+\tilde{\Lambda}_{j}^{i} \mathcal{T}_{k}^{j}\right) X_{ \pm}^{k} \pm d z^{N} \mathcal{T}_{j}^{i} X_{ \pm N}^{j}
$$

We wedge the plus equation by $d z^{+}$and minus equation by $d z^{-}$to get

$$
\begin{gathered}
d z^{+} \wedge d z^{-} \tilde{X}_{+-}=d z^{+} \wedge\left(d \mathcal{T}_{k}^{i}-\mathcal{T}_{j}^{i} \Lambda_{k}^{j}+\tilde{\Lambda}_{j}^{i} \mathcal{T}_{k}^{j}\right) X_{+}^{k}+d z^{+} \wedge d z^{-} \mathcal{T}_{j}^{i} X_{+-}^{j} \\
d z^{-} \wedge d z^{+} \tilde{X}_{-+}=-d z^{-} \wedge\left(d \mathcal{T}_{k}^{i}-\mathcal{T}_{j}^{i} \Lambda_{k}^{j}+\tilde{\Lambda}_{j}^{i} \mathcal{T}_{k}^{j}\right) X_{-}^{k}-d z^{-} \wedge d z^{+} \mathcal{T}_{j}^{i} X_{-+}^{j}
\end{gathered}
$$

we set left-hand sides equal to each other using $\tilde{X}_{+-}=-\tilde{X}_{-+}$and $d z^{+} \wedge$ $d z^{-}=-d z^{-} \wedge d z^{+}$. We notice that we have symmetric expression which has antisymmetric terms in pairs. Therefore expressions from (53) to (173) can be repeated. This ends up with the same result, curvatures of the supersymmetric sigma models will be constant and opposite to each other, yielding the dual symmetric spaces. 


\section{Pseudoduality in Super WZW Models}

At this point it is interesting to discuss the pseudoduality transformations on super WZW models [18]. The super WZW model has considerable interest in the context of conformal field theory. We use the superspace with coordinates $\left(\sigma^{+}, \sigma^{-}, \theta^{+}, \theta^{-}\right)$where $\sigma^{ \pm}$are the standard lightcone coordinates, and $\theta^{ \pm}$ are the real Grassmann numbers, with supercharges $Q_{ \pm}=\partial_{\theta^{ \pm}}-i \theta^{ \pm} \partial_{ \pm}$and supercovariant derivatives $D_{ \pm}=\partial_{\theta^{ \pm}}+i \theta^{ \pm} \partial_{ \pm}$. To define super WZW model we introduce the superfield $\mathcal{G}(\sigma, \theta)$ in $G$ with components as expanded by

$$
\mathcal{G}(\sigma, \theta)=g(\sigma)\left(1+i \theta^{+} \psi_{+}(\sigma)+i \theta^{-} \psi_{-}(\sigma)+i \theta^{+} \theta^{-} \chi(\sigma)\right)
$$

where the fermions $\psi_{ \pm}(\sigma)$ take values in $\mathbf{g}$, and are the superpartners of the group-valued fields $g(\sigma)$. The field $\chi(\sigma)$ is the auxiliary field. The lagrangian of the model can be written as

$$
\mathcal{L}=\frac{1}{2} \operatorname{Tr}\left(D_{+} \mathcal{G}^{-1} D_{-} \mathcal{G}\right)+\Gamma
$$

where $\boldsymbol{\Gamma}$ represents the WZ term. Equations of motion following from this lagrangian are

$$
\begin{aligned}
D_{-}\left(\mathcal{G}^{-1} D_{+} \mathcal{G}\right) & =0 \\
D_{+}\left[\left(D_{-} \mathcal{G}\right) \mathcal{G}^{-1}\right] & =0
\end{aligned}
$$

There is a global symmetry $G_{L} \times G_{R}$ which gives the conserved super currents $\mathcal{J}_{+}^{L}=\mathcal{G}^{-1} D_{+} \mathcal{G}$ and $\mathcal{J}_{-}^{R}=\left(D_{-} \mathcal{G}\right) \mathcal{G}^{-1}$.

We can write similar expressions related to pseudodual WZW model with tilde. One can write the pseudoduality transformations using the similarity with bosonic case

$$
\begin{aligned}
& \tilde{\mathcal{G}}^{-1} D_{+} \tilde{\mathcal{G}}=+\mathcal{T}(\sigma, \theta) \mathcal{G}^{-1} D_{+} \mathcal{G} \\
& \tilde{\mathcal{G}}^{-1} D_{-} \tilde{\mathcal{G}}=-\mathcal{T}(\sigma, \theta) \mathcal{G}^{-1} D_{-} \mathcal{G}
\end{aligned}
$$

Taking $D_{-}$of first equation (124) followed by (122) yields that $D_{-} \mathcal{T}(\sigma, \theta)=$ 0 . If $\mathcal{T}(\sigma, \theta)$ is expanded as $\mathcal{T}(\sigma, \theta)=T(\sigma)+\theta^{+} \lambda_{+}+\theta^{-} \lambda_{-}+\theta^{+} \theta^{-} N(\sigma)$, then the condition $D_{-} \mathcal{T}(\sigma, \theta)$ implies that $\lambda_{-}=0, N(\sigma)=0, \partial_{-} T(\sigma)=0$ and $\partial_{-} \lambda_{+}=0$. Hence $\mathcal{T}$ turns out to be

$$
\mathcal{T}(\sigma, \theta)=T\left(\sigma^{+}\right)+\theta^{+} \lambda_{+}\left(\sigma^{+}\right)
$$


Taking $D_{+}$of second equation (125) gives the following equation

$$
D_{+} \mathcal{T}_{j}^{j}(\sigma, \theta)=\left(\tilde{f}_{m n}^{i} \mathcal{T}_{j}^{m} \mathcal{T}_{k}^{n}-f_{j k}^{m} \mathcal{T}_{m}^{i}\right)\left(\mathcal{G}^{-1} D_{+} \mathcal{G}\right)^{k}
$$

Before going further to solve this equation, it is convenient to find out the values of some fields in terms of components. A brief computation shows that

$$
\begin{aligned}
\mathcal{G}^{-1} D_{+} \mathcal{G}= & i \psi_{+}+i \theta^{+}\left(g^{-1} \partial_{+} g-i \psi_{+}^{2}\right)+i \theta^{-}\left(\chi-i \psi_{-} \psi_{+}\right) \\
& -\theta^{+} \theta^{-}\left(\partial_{+} \psi_{-}+\left[g^{-1} \partial_{+} g, \psi_{-}\right]+\left[\psi_{+}, \chi\right]\right) \\
\mathcal{G}^{-} D_{-} \mathcal{G}= & i \psi_{-}-i \theta^{+}\left(\chi-i \psi_{-} \psi_{+}\right)+i \theta^{-}\left(g^{-1} \partial_{-} g-i \psi_{-}^{2}\right) \\
& +\theta^{+} \theta^{-}\left(\partial_{-} \psi_{+}+\left[g^{-1} \partial_{-} g, \psi_{+}\right]+\left[\chi, \psi_{-}\right]\right) \\
\left(D_{-} \mathcal{G}\right) \mathcal{G}^{-1}= & g\left\{i \psi_{-}-i \theta^{+}\left(\chi-i \psi_{-} \psi_{+}\right)+i \theta^{-}\left(g^{-1} \partial_{-} g+i \psi_{-}^{2}\right)\right. \\
& +\theta^{+} \theta^{-}\left(\partial_{-} \psi_{+}+\left[\psi_{-}, \chi\right]\right\} g^{-1}
\end{aligned}
$$

Hence, the equation of motion (122) produces the following equations

$$
\begin{aligned}
\chi & =i \psi_{-} \psi_{+} \\
\partial_{-} \psi_{+} & =0 \\
\partial_{-}\left(g^{-1} \partial_{+} g-i \psi_{+}^{2}\right) & =0 \\
\partial_{+} \psi_{-} & =\left[\psi_{-}, g^{-1} \partial_{+} g\right]+\left[\chi, \psi_{+}\right]
\end{aligned}
$$

and (123) yields that

$$
\begin{aligned}
\chi & =i \psi_{-} \psi_{+} \\
\partial_{-} \psi_{+} & =\left[\chi, \psi_{-}\right] \\
\partial_{+} \psi_{-} & =\left[\psi_{-}, g^{-1} \partial_{+} g\right] \\
\partial_{+}\left(g^{-1} \partial_{-} g+i \psi_{-}^{2}\right) & =\left[g^{-1} \partial_{-} g+i \psi_{-}^{2}, g^{-1} \partial_{+} g\right]
\end{aligned}
$$

We see that (131) and (135) are the same expressions, and determines the auxiliary field in terms of $\psi_{-}$and $\psi_{+}$. (132) implies that $\psi_{+}$depends on $\sigma^{+}$only, and (136) points out that $\chi$ commutes with $\psi_{-}$as expected. (133) gives us the bosonic left current conservation law by means of (132). Comparison of (134) with (137) shows that $\chi$ commutes with $\psi_{+}$, and (137) is the fermionic equation of motion for $\psi_{-}$, which leads (138) to the bosonic right current conservation law. Finally we may eliminate $\psi_{ \pm}^{2}$ terms because these are fermionic fields and anticommute with each other. 
Therefore the fields (128)-(130) can be written in simplified forms as

$$
\begin{aligned}
\mathcal{G}^{-1} D_{+} \mathcal{G} & =i \psi_{+}+i \theta^{+} g^{-1} \partial_{+} g \\
\mathcal{G}^{-1} D_{-} \mathcal{G} & =i \psi_{-}+i \theta^{-} g^{-1} \partial_{-} g+\theta^{+} \theta^{-}\left[g^{-1} \partial_{-} g, \psi_{+}\right] \\
\left(D_{-} \mathcal{G}\right) \mathcal{G}^{-1} & =i g \psi_{-} g^{-1}+i \theta^{-}\left(\partial_{-} g\right) g^{-1}
\end{aligned}
$$

We can now solve the equation (127) using (126) and (139). A little computation gives the components of $\mathcal{T}(\sigma, \theta)$ as

$$
\begin{aligned}
\left(\lambda_{+}\right)_{j}^{i}= & i\left(\tilde{f}_{m n}^{i} T_{j}^{m} T_{k}^{n}-f_{j k}^{m} T_{m}^{i}\right) \psi_{+}^{k} \\
\left(\partial_{+} T\right)_{j}^{i}= & \left(\tilde{f}_{m n}^{i}\left(\lambda_{+}\right)_{j}^{m} T_{k}^{n}+\tilde{f}_{m n}^{i} T_{j}^{m}\left(\lambda_{+}\right)_{k}^{n}-f_{j k}^{m}\left(\lambda_{+}\right)_{m}^{i}\right) \psi_{+}^{k} \\
& +\left(\tilde{f}_{m n}^{i} T_{j}^{m} T_{k}^{n}-f_{j k}^{m} T_{m}^{i}\right)\left(g^{-1} \partial_{+} g\right)^{k}
\end{aligned}
$$

If (142) is inserted in (143) the result follows

$$
\begin{aligned}
\left(\partial_{+} T\right)_{j}^{i}= & \left(\tilde{f}_{m n}^{i} T_{j}^{m} T_{k}^{n}-f_{j k}^{m} T_{m}^{i}\right)\left(g^{-1} \partial_{+} g\right)^{k}-i \tilde{f}_{k l}^{i} \tilde{f}_{m n}^{l}\left(T_{j}^{m} T_{b}^{k}-T_{j}^{k} T_{b}^{m}\right) T_{a}^{n} \psi_{+}^{a} \psi_{+}^{b} \\
& -i \tilde{f}_{k l}^{i} f_{b a}^{m} T_{j}^{k} T_{m}^{l} \psi_{+}^{a} \psi_{+}^{b}+i f_{j b}^{m} f_{m a}^{n} T_{n}^{i} \psi_{+}^{a} \psi_{+}^{b}
\end{aligned}
$$

We want to find perturbation solution, and we notice that the order of the term $g^{-1} \partial_{+} g$ is proportional to the order of the term $\psi \psi$. We find the following perturbative result up to the second order terms after integrating (144)

$$
T_{j}^{i}\left(\sigma^{+}\right)=T_{j}^{i}(0)+A_{j k}^{i} \int_{0}^{\sigma^{+}}\left(g^{-1} \partial_{+} g\right)^{k} d \sigma^{\prime+}+B_{j a b}^{i} \int_{0}^{\sigma^{+}} \psi_{+}^{a} \psi_{+}^{b} d \sigma^{\prime+}+H . O .
$$

where $T_{j}^{i}(0)=\delta_{j}^{i}, A_{j k}^{i}=\left(\tilde{f}_{j k}^{i}-f_{j k}^{i}\right)$, and $B_{j a b}^{i}=i\left(\tilde{f}_{a k}^{i} \tilde{f}_{b j}^{k}+\tilde{f}_{j k}^{i} f_{a b}^{k}+f_{a k}^{i} f_{b j}^{k}\right)$. Therefore $\lambda_{+}$may be written as

$$
\begin{aligned}
\left(\lambda_{+}\right)_{j}^{i}= & i A_{j k}^{i} \psi_{+}^{k}+C_{j k c}^{i} \psi_{+}^{k} \int_{0}^{\sigma^{+}}\left(g^{-1} \partial_{+} g\right)^{c} d \sigma^{+} \\
& +i D_{j k c d}^{i} \psi_{+}^{k} \int_{0}^{\sigma^{+}} \psi_{+}^{c} \psi_{+}^{d} d \sigma^{\prime+}+\text { H.O. }
\end{aligned}
$$

where constants $C_{j k c}^{i}$ and $D_{j k c d}^{i}$ are

$$
\begin{aligned}
C_{j k c}^{i}= & \tilde{f}_{j n}^{i} A_{k c}^{n}+\tilde{f}_{n k}^{i} A_{j c}^{n}-f_{j k}^{n} A_{n c}^{i}=\left(\tilde{f}_{n c}^{i} \tilde{f}_{j k}^{n}-\tilde{f}_{n[j}^{i} f_{c k]}^{n}+f_{n c}^{i} f_{j k}^{n}\right) \\
D_{j k c d}^{i}= & \tilde{f}_{j n}^{i} B_{k c d}^{n}+\tilde{f}_{n k}^{i} B_{j c d}^{n}-f_{j k}^{n} B_{n c d}^{i}=i \tilde{f}_{j n}^{i} \tilde{f}_{c m}^{n} \tilde{f}_{k d}^{m}+i \tilde{f}_{n k}^{i} \tilde{f}_{c m}^{n} \tilde{f}_{j d}^{m} \\
& +i \tilde{f}_{j n}^{i} \tilde{f}_{k m}^{n} f_{d a}^{m}+i \tilde{f}_{n k}^{i} \tilde{f}_{j m}^{n} f_{d c}^{m}-i \tilde{f}_{c m}^{i} \tilde{f}_{n d}^{m} f_{j k}^{n}+i \tilde{f}_{j n}^{i} f_{c m}^{n} f_{k d}^{m} \\
& +i \tilde{f}_{n k}^{i} f_{c m}^{n} f_{j d}^{m}-i \tilde{f}_{n m}^{i} f_{d c}^{m} f_{j k}^{n}-i f_{c m}^{i} f_{n d}^{m} f_{j k}^{n}
\end{aligned}
$$


As seen we have an expression for the transformation matrix (126) up to the third order terms. We notice that $T$ represents even order terms while $\lambda_{+}$represents odd order terms. Now we can proceed to find expressions on $\tilde{\mathbb{M}}$ using pseudoduality equations (124) and (125). If (126) and (139) are substituted in the first equation we obtain

$$
\begin{aligned}
\tilde{\psi}_{+}^{i} & =T_{j}^{i} \psi_{+}^{j} \\
\left(\tilde{g}^{-1} \partial_{+} \tilde{g}\right)^{i} & =T_{j}^{i}\left(g^{-1} \partial_{+} g\right)^{j}+\left(\lambda_{+}\right)_{j}^{i} \psi_{+}^{j}
\end{aligned}
$$

We notice that both of these equations depend only on $\sigma^{+}$. Likewise inserting (126) and (140) into second equation (125) leads to

$$
\begin{aligned}
\left(\lambda_{+}\right)_{j}^{i} \psi_{-}^{j} & =0 \\
\tilde{\psi}_{-}^{i} & =-T_{j}^{i} \psi_{-}^{j} \\
\left(\tilde{g}^{-1} \partial_{-} \tilde{g}\right)^{i} & =-T_{j}^{i}\left(g^{-1} \partial_{-} g\right)^{j} \\
{\left[\tilde{g}^{-1} \partial_{-} \tilde{g}, \tilde{\psi}_{+}\right]^{i} } & =-T_{j}^{i}\left[g^{-1} \partial_{-} g, \psi_{+}\right]^{j}+i\left(\lambda_{+}\right)_{j}^{i}\left(g^{-1} \partial_{-} g\right)^{j}
\end{aligned}
$$

These are the pseudoduality equations in components. We observe that if $\psi_{-}$and $\psi_{+}$are set to zero we obtain bosonic case pseudoduality equations as pointed out in ([6]). We see that the term $\left(\lambda_{+}\right)_{j}^{i} \psi_{+}^{j}$ in equation (150) gives us $\left(\lambda_{+}\right)_{j}^{i} \psi_{+}^{j}=-i\left[\tilde{\psi}_{+}, \tilde{\psi}_{+}\right]_{\tilde{G}}^{i}+i T_{j}^{i}\left[\psi_{+}, \psi_{+}\right]_{G}^{j}=0$. The last equation (154) gives us the constraint (142). The equation (151) is interesting because it tells us that $\left[\tilde{\psi}_{-}, \tilde{\psi}_{+}\right]^{i}=-T_{j}^{i}\left[\psi_{-}, \psi_{+}\right]^{j}$, which gives us two choices. First choice is $\lambda_{+}=0$ which leads to either

$$
\tilde{f}_{m n}^{i} T_{k}^{m} T_{l}^{n}=T_{j}^{i} f_{k l}^{j}
$$

if $\psi_{+} \neq 0$. This yields that $\partial_{+} T=0$ as can be seen from (143), and hence we get a trivial case, flat space pseudoduality equations as follows

$$
\begin{aligned}
\tilde{\psi}_{ \pm}^{i} & = \pm \psi_{ \pm}^{i} \\
\left(\tilde{g}^{-1} \partial_{ \pm} \tilde{g}\right)^{i} & = \pm\left(g^{-1} \partial_{ \pm} g\right)^{i}
\end{aligned}
$$

where we choose $T$ to be identity. Therefore we obtain $\tilde{f}_{j k}^{i}=f_{j k}^{i}$ in (155). Or we set $\psi_{+}=0$, and hence last term in (145) will be eliminated, so pseudoduality relations will be

$$
\begin{aligned}
\tilde{\psi}_{-}^{i}= & -\psi_{-}^{i}-\left[\psi_{-}, \int_{0}^{\sigma^{+}}\left(g^{-1} \partial_{+} g\right) d \sigma^{+}\right]_{\tilde{G}}^{i} \\
& +\left[\psi_{-}, \int_{0}^{\sigma^{+}}\left(g^{-1} \partial_{+} g\right) d \sigma^{+}\right]_{G}^{i}+\text { H.O. }
\end{aligned}
$$




$$
\begin{aligned}
\left(\tilde{g}^{-1} \partial_{ \pm} \tilde{g}\right)^{i}= & \pm\left(g^{-1} \partial_{ \pm} g\right)^{i} \pm\left[g^{-1} \partial_{ \pm} g, \int_{0}^{\sigma^{+}}\left(g^{-1} \partial_{+} g\right) d \sigma^{\prime+}\right]_{\tilde{G}}^{i} \\
& \mp\left[g^{-1} \partial_{ \pm} g, \int_{0}^{\sigma^{+}}\left(g^{-1} \partial_{+} g\right) d \sigma^{\prime+}\right]_{G}^{i}+\text { H.O. }
\end{aligned}
$$

where we introduced the bracket $[,]_{G / \tilde{G}}$ to represent the commutations in $G / \tilde{G}$. Second choice will eliminate $\psi_{-}$and hence we get whole expressions (145) and (146) for $T$ and $\lambda_{+}$. Therefore we obtain the following perturbation fields

$$
\begin{aligned}
\tilde{\psi}_{+}^{i}= & \psi_{+}^{i}+\left[\psi_{+}, \int_{0}^{\sigma^{+}}\left(g^{-1} \partial_{+} g\right) d \sigma^{\prime+}\right]_{\tilde{G}}^{i}-\left[\psi_{+}, \int_{0}^{\sigma^{+}}\left(g^{-1} \partial_{+} g\right) d \sigma^{\prime+}\right]_{G}^{i} \\
& +i \int_{0}^{\sigma^{+}}\left[\psi_{+}\left(\sigma^{\prime+}\right),\left[\psi_{+}\left(\sigma^{\prime+}\right), \psi_{+}\left(\sigma^{+}\right)\right]_{\tilde{G}}\right]_{\tilde{G}}^{i} d \sigma^{\prime+} \\
& +i \int_{0}^{\sigma^{+}}\left[\psi_{+}\left(\sigma^{++}\right),\left[\psi_{+}\left(\sigma^{\prime+}\right), \psi_{+}\left(\sigma^{+}\right)\right]_{G}\right]_{G}^{i} d \sigma^{\prime+}+H . O . \\
\left(\tilde{g}^{-1} \partial_{ \pm} \tilde{g}\right)^{i}= & \pm\left(g^{-1} \partial_{ \pm} g\right)^{i} \pm\left[g^{-1} \partial_{ \pm} g, \int_{0}^{\sigma^{+}}\left(g^{-1} \partial_{+} g\right) d \sigma^{\prime+}\right]_{\tilde{G}}^{i} \mp\left[g^{-1} \partial_{ \pm} g, \int_{0}^{\sigma^{+}}\left(g^{-1} \partial_{+} g\right) d \sigma^{\prime+}\right]_{G}^{i} \\
& \pm i \int_{0}^{\sigma^{+}}\left[\psi_{+}\left(\sigma^{\prime+}\right),\left[\psi_{+}\left(\sigma^{++}\right),\left(g^{-1} \partial_{ \pm} g\right)\left(\sigma^{+}\right)\right]_{\tilde{G}}\right]_{\tilde{G}}^{i} d \sigma^{\prime+} \\
& \pm i \int_{0}^{\sigma^{+}}\left[\psi_{+}\left(\sigma^{\prime+}\right),\left[\psi_{+}\left(\sigma^{\prime+}\right),\left(g^{-1} \partial_{ \pm} g\right)\left(\sigma^{+}\right)\right]_{G}\right]_{G}^{i} d \sigma^{\prime+}+H . O .
\end{aligned}
$$

where the cross terms $\left[,[,]_{G}\right]_{\tilde{G}}$ vanish.

We have already derived our pseudoduality equations, conditions inducing pseudoduality, and finally the perturbative expressions of the pseudodual fields up to the third (fourth) order terms, leading to conserved currents on the pseudodual model. Using these fields it is possible to construct left and right super currents on pseudodual manifold $\tilde{G}$. It is apparent from the expression (139) that we can easily construct right super currents belonging to special cases discussed above. To find left super currents we use the method we traced in [19, 20]. 


\subsection{Supercurrents in Flat Space Pseudoduality}

In this case structure constants of both models are the same, $\tilde{f}=f$, and pseudoduality relations are given by (156) and (157). We let $g=e^{Y}$, where $Y$ is the lie algebra. Using the expansion [21, 22, 23, 20]

$$
g^{-1} \partial_{ \pm} g=\frac{1-e^{-a d Y}}{a d Y} \partial_{ \pm}=\sum_{k=0}^{\infty} \frac{(-1)^{k}}{(k+1) !}\left[Y, \ldots,\left[Y, \partial_{ \pm} Y\right]\right]
$$

where $a d Y$ is the adjoint representation of $Y$, and $\operatorname{ad} Y(Z)=[Y, Z]$. We know that bosonic currents are invariant under $g \longrightarrow g_{R}\left(\sigma^{-}\right) g_{L}\left(\sigma^{+}\right)$, hence we obtain that $g^{-1} \partial_{+} g \longrightarrow g_{L}^{-1} \partial_{+} g_{L}$, which is

$$
g_{L}^{-1} \partial_{+} g_{L}=\partial_{+} Y_{L}-\frac{1}{2 !}\left[Y_{L}, \partial_{+} Y_{L}\right]+\frac{1}{3 !}\left[Y_{L},\left[Y_{L}, \partial_{+} Y_{L}\right]\right]+\ldots
$$

Now we impose that $Y=\sum_{0}^{\infty} \varepsilon^{n} y_{n}$, where $\varepsilon$ is a small parameter. Thus we get the following lie algebra valued field up to the third order terms

$$
\begin{aligned}
g_{L}^{-1} \partial_{+} g_{L}= & \varepsilon \partial_{+} y_{L 1}+\varepsilon^{2}\left(\partial_{+} y_{L 2}-\frac{1}{2}\left[y_{L 1}, \partial_{+} y_{L 1}\right]\right) \\
& +\varepsilon^{3}\left(\partial_{+} y_{L 3}-\frac{1}{2}\left[y_{L 1}, \partial_{+} y_{L 2}\right]-\frac{1}{2}\left[y_{L 2}, \partial_{L 1}\right]+\frac{1}{6}\left[y_{L 1},\left[y_{L 1}, \partial_{+} y_{L 1}\right]\right]\right)+\mathcal{O}\left(\varepsilon^{4}\right)
\end{aligned}
$$

In a similar way one can find the expression for $g^{-1} \partial_{-} g$ [20]

$$
\begin{aligned}
g^{-1} \partial_{-} g= & \varepsilon \partial_{-} y_{R 1}+\varepsilon^{2}\left(\partial_{-} y_{R 2}-\left[y_{L 1}, \partial_{-} y_{R 1}\right]-\frac{1}{2}\left[y_{R 1}, \partial_{-} y_{R 1}\right]\right) \\
& +\varepsilon^{3}\left(\partial_{-} y_{R 3}-\left[y_{L 2}, \partial_{-} y_{R 1}\right]-\left[y_{L 1}, \partial_{-} y_{R 2}\right]-\frac{1}{2}\left[y_{R 2}, \partial_{-} y_{R 1}\right]-\frac{1}{2}\left[y_{R 1}, \partial_{-} y_{R 2}\right]\right) \\
& +\frac{1}{2}\left[y_{L 1},\left[y_{R 1}, \partial_{-} y_{R 1}\right]\right]+\frac{1}{2}\left[y_{L 1},\left[y_{L 1}, \partial_{-} y_{R 1}\right]\right]+\mathcal{O}\left(\varepsilon^{4}\right)
\end{aligned}
$$

Since it works all the way up we are going to do all our calculations up to the second order of $\varepsilon$ for simplicity and demonstration. We can write similar expressions for the manifold $\tilde{G}$. Pseudoduality equation (157) gives infinite number of sub-pseudoduality equations, from which we may write 
the following expressions coming from up to the second order of $\varepsilon$ terms

$$
\begin{aligned}
\partial_{+} \tilde{y}_{L 1} & =\partial_{+} y_{L 1} \\
\partial_{-} \tilde{y}_{R 1} & =-\partial_{-} y_{R 1} \\
\partial_{+} \tilde{y}_{L 2}-\frac{1}{2}\left[\tilde{y}_{L 1}, \partial_{+} \tilde{y}_{L 1}\right] & =\partial_{+} y_{L 2}-\frac{1}{2}\left[y_{L 1}, \partial_{+} y_{L 1}\right] \\
\partial_{-} \tilde{y}_{R 2}-\left[\tilde{y}_{L 1}, \partial_{-} \tilde{y}_{R 1}\right]-\frac{1}{2}\left[\tilde{y}_{R 1}, \partial_{-} \tilde{y}_{R 1}\right] & =-\partial_{-} y_{R 2}+\left[y_{L 1}, \partial_{-} y_{R 1}\right]+\frac{1}{2}\left[y_{R 1}, \partial_{-} y_{R 1}\right]
\end{aligned}
$$

First equation yields that $\tilde{y}_{L 1}=y_{L 1}+C_{L 1}$, where $C_{L 1}$ is constant, and the second equation gives $\tilde{y}_{R 1}=-y_{R 1}-C_{R 1}$, where $C_{R 1}$ is constant. Inserting these result into last equation gives

$$
\partial_{-} \tilde{y}_{R 2}+\frac{1}{2}\left[\tilde{y}_{R 1}, \partial_{-} \tilde{y}_{R 1}\right]=-\partial_{-} y_{R 2}+\frac{3}{2}\left[y_{R 1}, \partial_{-} y_{R 1}\right]
$$

where we used the equality of structure constants. We found this because we need this term in the expansion of bosonic right current 3 , which is

$$
\left(\partial_{-} g_{R}\right) g_{R}^{-1}=\varepsilon \partial_{-} y_{R 1}+\varepsilon^{2}\left(\partial_{-} y_{R 2}+\frac{1}{2}\left[y_{R 1}, \partial_{-} y_{R 1}\right]\right)+\mathcal{O}\left(\varepsilon^{3}\right)
$$

Hence bosonic right and left currents on $\tilde{G}$ in terms of nonlocal expressions will be

$$
\begin{aligned}
& \tilde{J}_{+}^{L}=\tilde{g}_{L}^{-1} \partial_{+} \tilde{g}_{L}=\varepsilon \partial_{+} y_{L 1}+\varepsilon^{2}\left(\partial_{+} y_{L 2}-\frac{1}{2}\left[y_{L 1}, \partial_{+} y_{L 1}\right]\right)+\mathcal{O}\left(\varepsilon^{3}\right) \\
& \tilde{J}_{-}^{R}=\left(\partial_{-} \tilde{g}_{R}\right) \tilde{g}_{R}^{-1}=-\varepsilon \partial_{-} y_{R 1}-\varepsilon^{2}\left(\partial_{-} y_{R 2}-\frac{3}{2}\left[y_{R 1}, \partial_{-} y_{R 1}\right]\right)+\mathcal{O}\left(\varepsilon^{3}\right)
\end{aligned}
$$

Obviously these currents are conserved by means of (133) and (138). Now we consider the fermionic components, and we let $\psi_{ \pm}=\sum_{n=1}^{\infty} \varepsilon^{n} \psi_{n \pm}$. We denote $\psi_{ \pm}$as the sum of right and left components $\psi_{ \pm}=\psi_{R \pm}\left(\sigma^{-}\right)+\psi_{L \pm}\left(\sigma^{+}\right)$. But from (132) we understand that $\psi_{+}$includes $\psi_{L+}$ only. Pseudoduality relations (156) again yields infinite number of subequations

$$
\begin{aligned}
\tilde{\psi}_{L n+} & =\psi_{L n+} \\
\tilde{\psi}_{(L / R) n-} & =-\psi_{(L / R) n-}
\end{aligned}
$$

\footnotetext{
${ }^{3}$ see $([20])[21,22,23]$ for details of this expansion
} 
which hold true for each $n$. Thus left and right supercurrents on $\tilde{G}$ in nonlocal terms up to the second order of $\varepsilon$ will be

$$
\begin{gathered}
\tilde{\mathcal{J}}_{+}^{L}=\tilde{\mathcal{G}}^{-1} D_{+} \tilde{\mathcal{G}}=i \tilde{\psi}_{+}+i \theta^{+}\left(\tilde{g}^{-1} \partial_{+} \tilde{g}\right) \\
=i \varepsilon\left(\psi_{L 1+}+\theta^{+} \partial_{+} y_{L 1}\right)+i \varepsilon^{2}\left\{\psi_{L 2+}+\theta^{+}\left(\partial_{+} y_{L 2}-\frac{1}{2}\left[y_{L 1}, \partial_{+} y_{L 1}\right]\right)\right\}+\mathcal{O}\left(\varepsilon^{3}\right) \\
\tilde{\mathcal{J}}_{-}^{R}=\left(D_{-} \tilde{\mathcal{G}}\right) \tilde{\mathcal{G}}^{-1}=i \tilde{g} \tilde{\psi}_{-} \tilde{g}^{-1}+i \theta^{-}\left(\partial_{-} \tilde{g}\right) \tilde{g}^{-1} \\
=-i \varepsilon\left(\psi_{1-}+\theta^{-} \partial_{-} y_{R 1}\right)-i \varepsilon^{2}\left\{\psi_{2-}+\left[y_{L 1}, \psi_{1-}\right]-\left[y_{R 1}, \psi_{1-}\right]\right. \\
\left.\quad+\theta^{-}\left(\partial_{-} y_{R 2}-\frac{3}{2}\left[y_{R 1}, \partial_{-} y_{R 1}\right]\right)\right\}+\mathcal{O}\left(\varepsilon^{3}\right)
\end{gathered}
$$

It is obvious from the equations of motion that these currents in nonlocal expressions are conserved.

\subsection{Supercurrents in Anti-chiral Pseudoduality}

Now we consider our second case where $\psi_{+}$vanishes. In this case we need to be careful when using bracket relations because structure constants are different. We have already found our nonlocal expressions in (158) and (159). We use the same expansions of lie algebra $Y$ and fermionic field $\psi_{-}$in the powers of $\varepsilon$ as used in the previous part. Therefore pseudoduality relations up to the second order of $\varepsilon$ yield the following equations

$$
\begin{gathered}
\tilde{\psi}_{1-}^{i}=-\psi_{1-}^{i} \\
\tilde{\psi}_{2-}^{i}=-\psi_{2-}^{i}-\left[\psi_{1-}, y_{L 1}\right]_{\tilde{G}}^{i}+\left[\psi_{1-}, y_{L 1}\right]_{G}^{i} \\
\partial_{+} \tilde{y}_{L 1}^{i}=\partial_{+} y_{L 1}^{i} \\
\partial_{+} \tilde{y}_{L 2}^{i}-\left[\tilde{y}_{L 1}, \partial_{+} \tilde{y}_{L 1}\right]_{\tilde{G}}^{i}=\partial_{+} y_{L 2}^{i}+\frac{1}{2}\left[y_{L 1}, \partial_{+} y_{L 1}\right]_{G}^{i}-\left[y_{L 1}, \partial_{+} y_{L 1}\right]_{\tilde{G}}^{i} \\
\partial_{-} \tilde{y}_{R 1}^{i}=-\partial_{-} y_{R 1}^{i} \\
\partial_{-} \tilde{y}_{R 2}^{i}+\frac{1}{2}\left[\tilde{y}_{R 1}, \partial_{-} \tilde{y}_{R 1}\right]_{\tilde{G}}^{i}=-\partial_{-} y_{R 2}^{i}+\frac{1}{2}\left[y_{R 1}, \partial_{-} y_{R 1}\right]_{G}^{i}+\left[y_{R 1}, \partial_{-} y_{R 1}\right]_{\tilde{G}}^{i}
\end{gathered}
$$


We may find out nonlocal supercurrents on the pseudodual manifold using these expressions

$$
\begin{aligned}
\tilde{\mathcal{J}}_{+}^{L} & =i \varepsilon \theta^{+} \partial_{+} y_{L 1}+i \varepsilon^{2} \theta^{+}\left\{\partial_{+} y_{L 2}+\frac{1}{2}\left[y_{L 1}, \partial_{+} y_{L 1}\right]_{G}^{i}-\left[y_{L 1}, \partial_{+} y_{L 1}\right]_{\tilde{G}}^{i}\right\}+\mathcal{O}\left(\varepsilon^{3}\right) \\
\tilde{\mathcal{J}}_{-}^{R} & =-i \varepsilon\left(\psi_{1-}+\theta^{-} \partial_{-} y_{R 1}\right)-i \varepsilon^{2}\left\{\psi_{2-}+\left[y_{L 1}, \psi_{1-}\right]_{G}-\left[y_{R 1}, \psi_{1-}\right]_{\tilde{G}}\right. \\
& \left.+\theta^{-}\left(\partial_{-} y_{R 2}-\frac{1}{2}\left[y_{R 1}, \partial_{-} y_{R 1}\right]_{G}-\left[y_{R 1}, \partial_{-} y_{R 1}\right]_{\tilde{G}}\right)\right\}+\mathcal{O}\left(\varepsilon^{3}\right)
\end{aligned}
$$

Obviously these currents in nonlocal expressions are conserved provided that equations of motion are satisfied.

\subsection{Supercurrents in Chiral Pseudoduality}

We consider our final case where $\psi_{-}$disappears. We notice that there is a contribution of chiral part in the isometry $T$ which leads to third order terms in the field expressions on the target space of pseudodual manifold as can be seen from equations (160) and (161). Again we keep in our minds that structure constants are different. If the same conventions for $Y$ and $\psi_{+}$are used as above, then pseudoduality relations up to the second order of $\varepsilon$ can be calculated. Expressions for the fields $\tilde{g}^{-1} \partial_{ \pm} \tilde{g}$ are the same as (180)-(183), and expression for the chiral field (160) gives that

$$
\begin{aligned}
\tilde{\psi}_{L 1+}^{i} & =\psi_{L 1+}^{i} \\
\tilde{\psi}_{L 2+}^{i} & =\psi_{L 2+}^{i}+\left[\psi_{L 1+}, y_{L 1}\right]_{\tilde{G}}^{i}-\left[\psi_{L 1+}, y_{L 1}\right]_{G}^{i}
\end{aligned}
$$

Then nonlocal conserved supercurrents are found to be

$$
\begin{aligned}
\tilde{\mathcal{J}}_{+}^{L} & =i \varepsilon\left(\psi_{L 1+}+\theta^{+} \partial_{+} y_{L 1}\right)+i \varepsilon^{2}\left\{\psi_{L 2+}+\theta^{+}\left(\partial_{+} y_{L 2}+\frac{1}{2}\left[y_{L 1}, \partial_{+} y_{L 2}\right]_{G}\right.\right. \\
& \left.\left.-\left[y_{L 1}, \partial_{+} y_{L 1}\right]_{\tilde{G}}\right)\right\}+\mathcal{O}\left(\varepsilon^{3}\right) \\
\tilde{\mathcal{J}}_{-}^{R} & =-i \varepsilon \theta^{-} \partial_{-} y_{R 1}-i \varepsilon^{2} \theta^{-}\left(\partial_{-} y_{R 2}-\frac{1}{2}\left[y_{R 1}, \partial_{-} y_{R 1}\right]_{G}-\left[y_{R 1}, \partial_{-} y_{R 1}\right]_{\tilde{G}}\right)+\mathcal{O}\left(\varepsilon^{3}\right)
\end{aligned}
$$

It is noted that all these supercurrents are the complements of each other, and special cases of a more general one. Under the limiting conditions they 
are equal to each other. If we denote the bosonic and fermionic components by $\tilde{J}_{B}$ and $\tilde{J}_{F}$ then they are written as

$$
\tilde{\mathcal{J}}_{ \pm}^{L / R}= \pm \tilde{J}_{F}^{L / R} \pm \theta^{ \pm} \tilde{J}_{B}^{L / R}
$$

Since these super currents serve as the orthonormal frame on the pullback bundle of the target space of $G$, we may find the corresponding bosonic and fermionic curvatures using them. If $L^{i}=\mathcal{J}^{i}$ is the left invariant Cartan one form which satisfies the Maurer-Cartan equation

$$
d \mathcal{J}^{i}+\frac{1}{2} f_{j k}^{i} \mathcal{J}^{j} \wedge \mathcal{J}^{k}=0
$$

and $L_{k}^{i}=\frac{1}{2} f_{j k}^{i} \mathcal{J}^{j}$ is the antisymmetric riemannian connection, then Cartan structural equations on superspace can be written as

$$
\begin{aligned}
& d L^{i}+L_{j}^{i} \wedge L^{j}=0 \\
& d L_{j}^{i}+L_{k}^{i} \wedge L_{j}^{k}=\frac{1}{2} \mathcal{R}_{j k l}^{i} L^{k} \wedge L^{l}
\end{aligned}
$$

where $\mathcal{R}_{j k l}^{i}$ is the curvature of superspace. If the calculations in the previous section is repeated using these equations in this case one can show that curvatures on $S O(G)$ and $S O(\tilde{G})$ are constants, and related to each other by $\tilde{\mathcal{R}}_{j k l}^{i}=-\mathcal{R}_{j k l}^{i}$, which shows that two superspaces are dual symmetric spaces. If this curvature relation is split into bosonic and fermionic parts, it is easy to see that fermionic part will yield a curvature relation which are opposite to each other, i.e. $\left(\tilde{\mathcal{R}}_{F}\right)_{j k l}^{i}=-\left(\mathcal{R}_{F}\right)_{j k l}^{i}$, while bosonic part will give that both curvatures will be the same, i.e. $\left(\tilde{\mathcal{R}}_{B}\right)_{j k l}^{i}=\left(\mathcal{R}_{B}\right)_{j k l}^{i}$, because of anticommuting numbers. This is consistent with the results found in the component expansion methods.

\section{Discussion}

We analyzed the pseudoduality conditions on the supersymmetric extensions of sigma models in two respects, by component expansion and orthonormal coframe method. In the first case we have seen that pseudoduality transformation in $\mathrm{N}=1$ supersymmetric sigma models imposes the condition that pseudoduality maps all points in the first manifold to only one point at which riemann normal coordinates are used on the pseudodual manifold. Although 
torsions of both models vanish in $(1,1)$ case, torsion of the pseudodual manifold exists in $(1,0)$ case. Curvatures when splitted to bosonic and fermionic parts yield that bosonic curvatures must be the same because of anticommuting grassmann numbers. It is obvious that pseudoduality transformation is not invertible if we would like to preserve these conditions unchanged on both manifolds. The only condition for invertibility of pseudoduality is that pseudoduality is between riemann normal coordinates with vanishing torsions. In the orthonormal coframe method we have seen that our results are similar to ones found before [6, 20]. When we consider the sigma models based on the Lie groups, we have seen that pseudoduality in components imposes three different conditions; flat space pseudoduality which yield that both $\lambda_{+}$and $T$ vanish, and structures constants are the same, (anti)chiral pseudoduality which yields $\left(\psi_{-}=0\right) \psi_{+}=0$ with distinct structure constants. These conditions are the result of equation (151). For each case we found the conserved super currents. We are going to use these results to find out the pseudoduality conditions and conserved currents when applied to symmetric spaces [24].

\section{Acknowledgments}

I would like to thank O. Alvarez for his comments, helpful discussions, and reading an earlier draft of the manuscript. I would like to thank E. A. Ivanov for bringing his important paper to my attention.

\section{References}

[1] S. V. Ketov, Quantum Non-Linear Sigma-Models: From Quantum Field Theory to Supersymmetry, Conformal Field Theory, Black Holes and Strings, Texts and Monographs in Physics, Springer, Berlin, Germany, 2000 .

[2] C.M. Hull and E. Witten, Supersymmetric sigma models and the heterotic string, Phys. Lett. B160 (1985) 398-402.

[3] T. Curtright and C.Zachos, Currents, charges, and canonical structure of pseudochiral models, Phys. Rev. D49 (1994) 5408-5421, hep-th/9401006/. 
[4] E. A. Ivanov, Duality in $d=2$ sigma models of chiral field with anomaly, Theor. Math. Phys. 71 (1987) 474-484.

[5] O. Alvarez, Target space pseudoduality between dual symmetric spaces, Nucl. Phys. B582 (2000) 139, hep-th/0004120/.

[6] O. Alvarez, Pseudoduality in Sigma Models, Nucl.Phys. B638 (2002) 328350, hep-th/0204011/.

[7] J. Wess and J. Bagger, Supersymmetry and Supergravity, Princeton Univ. Press, 1983.

[8] P.C. West, Introduction to Supersymmetry and Supergravity, World Scientific, 1986.

[9] S.J. Gates Jr., M.T. Grisaru, M. Roček, W. Siegel, Superspace or One Thousand and One Lessons in Supersymmetry, Benjamin/Cummings Publ., 1983.

[10] P. Freund, Introduction to Supersymmetry, Campridge Univ. Press, 1986.

[11] O. S. Zandron, The Heterotic Supersymmetric Sigma Model in the Canonical Exterior Formalism, International Journal of Theoretical Physics, Volume 46, Number 11 / November, 2007, 2758-2773.

[12] F. Delduc, S. Kalitzin and E. Sokatchev, Geometry of sigma -models with heterotic supersymmetry, 1990 Class. Quantum Grav. 7 1567-1582.

[13] G. Michalogiorgakis, S. S. Gubser, Heterotic non-linear sigma models with anti-de Sitter target spaces, Nucl.Phys.B757:146-171,2006.

[14] C. M. Hull, Gauged Heterotic Sigma-Models, Mod.Phys.Lett. A9 (1994) 161-168.

[15] S. Carroll, Spacetime and Geometry: An Introduction to General Relativity, Addison-Wesley, 2003.

[16] A. Hatzinikitas, A note on Riemann normal coordinates, hep-th/0001078/. 
[17] Michael B. Green, John H. Schwarz, Edward Witten, Superstring Theory, in 2 vols., Cambridge Univ. Press, 1987.

[18] J. M. Evans, M. Hassan, N. J. MacKay, and A. J. Mountain, Conserved charges and supersymmetry in principal chiral and WZW models, Nucl.Phys. B580 (2000) 605-646, hep-th/0001222/.

[19] M.Sarisaman, Pseudoduality and Conserved Currents in Sigma Models, Mod. Phys. Lett. A, Vol. 24, No. 2 (2009) pp. 123-134.

[20] M. Sarisaman, Pseudoduality Between Symmetric Space Sigma Models, hep-th/0904.4087/.

[21] S. Helgason, Differential Geometry, Lie Groups, and Symmetric Spaces, Academic Press, 1978.

[22] H. Eichenherr, M. Forger, Higher local conservation laws for nonlinear sigma models on symmetric spaces, Commun. Math. Phys. 82 (1981) 227.

[23] H. Eichenherr, M. Forger, More about non-linear sigma models on symmetric spaces, Nucl. Phys. B 164 (1980) 528.

[24] M.Sarisaman, Pseudoduality In Supersymmetric Sigma Models on Symmetric Spaces, hep-th/0904.4671/. 